\title{
State Modelling of the Land Mobile Propagation Channel with Multiple Satellites
}

\author{
Daniel Arndt, ${ }^{1}$ Alexander Ihlow, ${ }^{1}$ Albert Heuberger, ${ }^{2}$ and Ernst Eberlein ${ }^{2}$ \\ ${ }^{1}$ Department of Electrical Engineering and Information Technology, Ilmenau University of Technology, 98693 Ilmenau, Germany \\ ${ }^{2}$ Communications Department, Fraunhofer Institute for Integrated Circuits (IIS), 91058 Erlangen, Germany
}

Correspondence should be addressed to Daniel Arndt, daniel.arndt@tu-ilmenau.de

Received 27 July 2012; Accepted 8 October 2012

Academic Editor: César Briso Rodríguez

Copyright (C) 2012 Daniel Arndt et al. This is an open access article distributed under the Creative Commons Attribution License, which permits unrestricted use, distribution, and reproduction in any medium, provided the original work is properly cited.

\begin{abstract}
We evaluate a new approach for multisatellite state modelling: the Master-Slave approach. By this concept slave satellites are modelled according to an existing master, whereas the correlation between multiple slaves is omitted. Master-Slave is therefore a generic name for a state modelling concept, for which different realisations are possible. As a possible realisation we present the Conditional Assembling Method. For modelling of only two satellites (one master and one slave), the Conditional Assembling Method enables an accurate resimulation of the correlation coefficient between the satellites and the probabilities of single and combined states. Based on this condition, the performance of Master-Slave for three, four, and five satellites is evaluated in terms of state probability modelling. Therefore, the correlation coefficients and the all bad-state probabilities with Master-Slave are compared with the measurements for different elevation angles and azimuth angle separations of the multisatellite system. Master-Slave has a high modelling error in case of small azimuth separation between the slave satellites (except that one slave has a small azimuth separation to the master). Furthermore, a master satellite with a high elevation provides a lower probability error compared to a master with low elevation.
\end{abstract}

\section{Introduction}

Satellites play an important role in today's commercial broadcasting systems. In cooperation with terrestrial repeaters they can ensure uninterrupted service of multimedia content (e.g., audio and video streaming) to stationary, portable, and mobile receivers. However, in case of mobile reception fading regularly disrupts the signal transmission due to shadowing or blocking objects between satellite and receiver. To mitigate these fading effects, diversity techniques such as angle diversity (multiple satellites) and time diversity (interleaving) are attractive. For link-level studies of the land mobile satellite (LMS) channel, statistical channel models are frequently used that are able to generate time series of the received fading signal [1-3].

Statistical LMS channel models describe several fading processes of the received signal: slow variations of the signal are caused by obstacles between the satellite and the receiver, which induce varying shadowing conditions of the direct signal component. Fast signal variations are caused by multipath effects due to static or moving scatterers in the vicinity of the mobile terminal. For short time periods these two components (slow and fast variations) are usually modelled by a stationary stochastic process, for example, as a Loo-distributed fading signal [1]. For longer time periods the received signal cannot be assumed as stationary. Therefore, statistical LMS channel models describe different receiving states to assess the large dynamic range of the received signal. The states (very slow variations) correspond to slowly varying environmental conditions (e.g., line of trees, buildings, zones with line-of-sight condition) in the transmission path. Figure 1 shows a state-of-theart LMS narrowband model for single-satellite reception according to Prieto-Cerdeira et al. [4]. It describes two states: a "good" state (corresponding to line-of-sight/light shadowing) and a "bad" state (corresponding to heavy shadowing/blockage). Within the states this model assumes a stationary Loo-distributed fading signal. It includes a slow fading component (lognormal fading) corresponding to varying shadowing conditions of the direct signal and a 


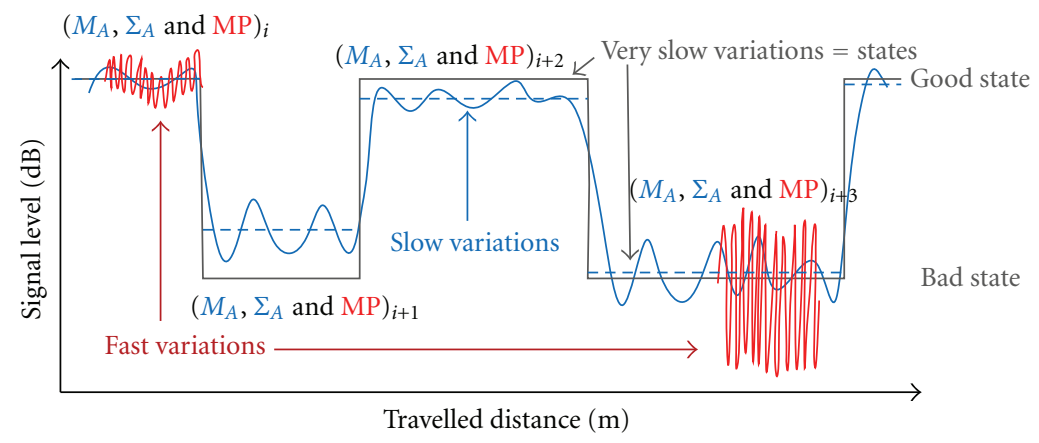

FIGURE 1: Signal components of a two-state LMS channel model.

fast fading component due to multipath effects. To enable a realistic modelling over the full dynamic range of the received signals, a random set of Loo parameters $\left(M_{A}, \Sigma_{A}\right.$, and MP) is generated after each state transition.

Focus of recent activities is the extension of this model for dual-satellite and multisatellite reception. It is realised by introducing correlation between states and between statistical parameters for fast and slow variations. However, the dominating part of the overall signal correlation stems from the state correlation.

Focus of this paper is state sequence modelling for multisatellite systems, assuming two states per satellite: "good" state and "bad" state. For this purpose, a new method for state sequence generation is introduced: the MasterSlave approach. Within Master-Slave it is assumed that each slave satellite depends only on a master satellite, whereas the correlation between different slaves is not described.

The paper is organised as follows: Section 2 gives an overview on state modelling approaches for single- and dual-satellite reception. In Section 3 the concept MasterSlave and a first implementation are introduced. Afterwards, in Section 4 a validation of Master-Slave for multisatellite reception in terms of state probability modelling is presented. Finally in Section 5 the conclusions are drawn.

\section{State Modelling for Single-Satellite and Dual-Satellite Reception}

In this section available state modelling approaches for single- and dual-satellite reception are summarised.

\subsection{Channel State Models for Single-Satellite Systems}

2.1.1. First-Order Markov Model. A Markov model is a special random process for generating discrete samples corresponding to channel states $s$ of a predefined sample length. For a first-order Markov model, each state depends only on the previous state. The conditional probabilities of state $s_{n+1}$ given the state $s_{n}$ are described by state transition probabilities $p_{i j}$. Therefore, the only parameter of the Markov chain is the state transition probability matrix (STPM) $\mathbf{P}_{\text {trans }} \in \mathbb{R}_{0+}^{N \times N}$ with $N$ being the number of states. The main characteristic of a first-order Markov chain is that it enables an exact modelling of the state probability and the average state duration. The state duration probability density function (SDPDF) of the first-order Markov chain follows an exponential distribution, which is found as not realistic for the LMS channel $[5,6]$. The first-order Markov model is used in early LMS models $[2,3]$.

2.1.2. Semi-Markov Model. To improve the state duration modelling for the LMS channel, semi-Markov chains were proposed in [5]. In contrast to the first-order Markov model, the state transitions do not occur at concrete time intervals. In fact, the time interval of the model staying in state $i$ depends directly on its SDPDF. As with the first-order Markov model, the state transitions are described with the state transition probability $p_{i j}$, but with $i \neq j$. Assuming a single-satellite model of only two states, the state transition probability is $p_{i j}=1$. The semi-Markov model allows some options to describe the SDPDF of each state, which defines the number of required parameters for the semi-Markov model. An overview is given in [7].

2.1.3. Dynamic Markov Model. A further method to improve the state duration modelling are dynamic Markov chains introduced in [6]. For dynamic Markov chains the state transition probability depends on the current state duration $n$ with $p_{i j}=f(n)$. Therefore, the two-dimensional STPM is extended to a three-dimensional state transition probability tensor (STPT) $\mathcal{P}_{\text {trans }} \in \mathbb{R}_{0+}^{N \times N \times n_{\max }}$, where $n_{\max }$ corresponds to the maximum state length. The dynamic Markov model enables an exact reproduction of the state probabilities and also an exact remodelling of the measured SDPDF. As a disadvantage, a high number of parameters are required to describe the STPT. Model approximations to reduce the number of parameters are presented in [6].

\subsection{Channel State Models for Dual-Satellite Systems}

2.2.1. Straightforward Method: Extension to a Multistate Model. The first-order Markov model, semi-Markov model, and the dynamic Markov model can be easily adapted for dual- or multisatellite modelling. This can be achieved by combining the single-satellite states "good" and "bad" from two satellites into joint states: "good good," "good bad," "bad 
good," and "bad bad". In case of the first-order Markov model, the $2 \times 2$ STPM becomes a $4 \times 4$ STPM. For the dynamic Markov model a $4 \times 4 \times n_{\max }$ STPT is required for the state series simulation. In case of the semi-Markov approach a $4 \times 4$ STPM and four separate state duration statistics are required for the dual-satellite modelling.

An obvious problem with this straightforward approach is the exponential growth of the number of parameters with the number of satellites. A multisatellite model has $N^{k}$ combined states with $N$ being the number of states per satellite and $k$ being the number of satellites.

2.2.2. Lutz Model. In [8] a state model for two correlated satellites based on first-order Markov chains was developed. This algorithm is based on first-order Markov chains and generates a joint STPM $(4 \times 4$ elements $)$ from two independent single-satellite STPMs (each with $2 \times 2$ elements), only by the use of one correlation coefficient. Using the joint STPM, a joint sequence of four combined states can be generated. The high flexibility of this algorithm becomes clear, since it requires only single-satellite parameter sets that are easy to derive from measurements and are already available in the literature for different elevation angles and a high number of environments. Databases for correlation coefficients are available as well for different environments, elevation angles, and angular separations of the azimuth and elevation angles. In contrast to this Lutz model, the above mentioned straightforward methods need complete datasets for any combination of elevation angles, azimuth angle separations, and environments to achieve the same variability. The Lutz model accurately resimulates the state probabilities of each single satellite as well as of the combined states. A limitation is that there is no flexibility in describing the state duration distribution. Furthermore, the current approach in [8] is limited to the usage of two satellites with two states per satellite.

\section{State Modelling for Multisatellite Reception: The Master-Slave Approach}

In general, all state models mentioned in the previous section are extendable to multiple satellites by assuming a multistate model with combined states. However, the number of required parameters for these models grows exponentially with the number of satellites. In practise, already the description of a satellite system with two satellites is challenging [7]. To avoid excessive complexity of a state model parametrisation with more than two satellites, the Master-Slave approach was proposed in [9]. Within the Master-Slave approach it is assumed that each slave satellite depends only on a master satellite, whereas the correlation between the slaves is not described.

Figure 2 demonstrates the Master-Slave approach with four satellites. Within Master-Slave only three satellite links in terms of correlation coefficients are described therefore. A full description of a four-satellite system would require the description of six correlations. In case of $k$ satellites the Master-Slave approach describes $k-1$ satellite links, whereas

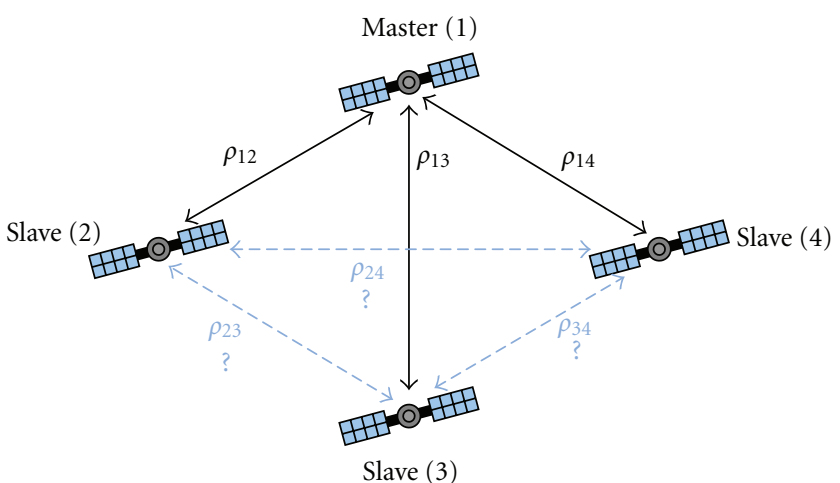

FIGURE 2: Master-Slave approach for multisatellite modelling. Several slave satellites are modelled according to the correlation to one master satellite, while neglecting the correlation between the slave satellites. The Master-Slave method has a reduced complexity compared to the conventional approach, where each individual correlation is described.

the full description requires $k \cdot(k-1) / 2$ links. As a result, Master-Slave reduces the complexity of a $k$-satellite model by $2 / k$.

It is not surprising that the missing correlation branches between the slaves could be a reduction of relevant information in case of multisatellite modelling. Therefore, a performance analysis of Master-Slave for multisatellite modelling (with at least three satellites) is found in Section 4. A first realisation of Master-Slave is described in Section 3.1.

3.1. First Realisation of State Modelling with Master-Slave: Conditional Assembling Method. The Master-Slave approach in its simplest form can be reduced to a two-satellite modelling problem, that is, the modelling of the master and one slave. The challenge within Master-Slave is the generation of a slave state sequence based on an existing master state sequence by providing, for example, a certain state probability and a correlation coefficient between the satellites. For this purpose, the conditional state sequences of the slave in case of a constant master state have to be analysed.

According to this condition, various realisations of Master-Slave are possible. Thus, the term Master-Slave describes a concept-not a concrete implementation.

In the following we present one possible realisation of Master-Slave that we define as Conditional Assembling Method (cf. Figure 3). For parametrisation, the principle of the Conditional Assembling Method is first to concatenate all parts of the slave state sequence for which the master is in "good" state. Afterwards, this state sequence is parametrised following an arbitrary Markov model (e.g., first-order Markov, semi-Markov). Same procedure is done for the slave state sequence in case master is in "bad" state.

The simulation is done in reversed order. First, the master state sequence is modelled. Second, two conditional slave state sequences with respect to the master state are generated independently (i.e., the conditional slave sequence for master is "good," and the conditional slave sequence for master is "bad"). For master and conditional slave states an arbitrary 


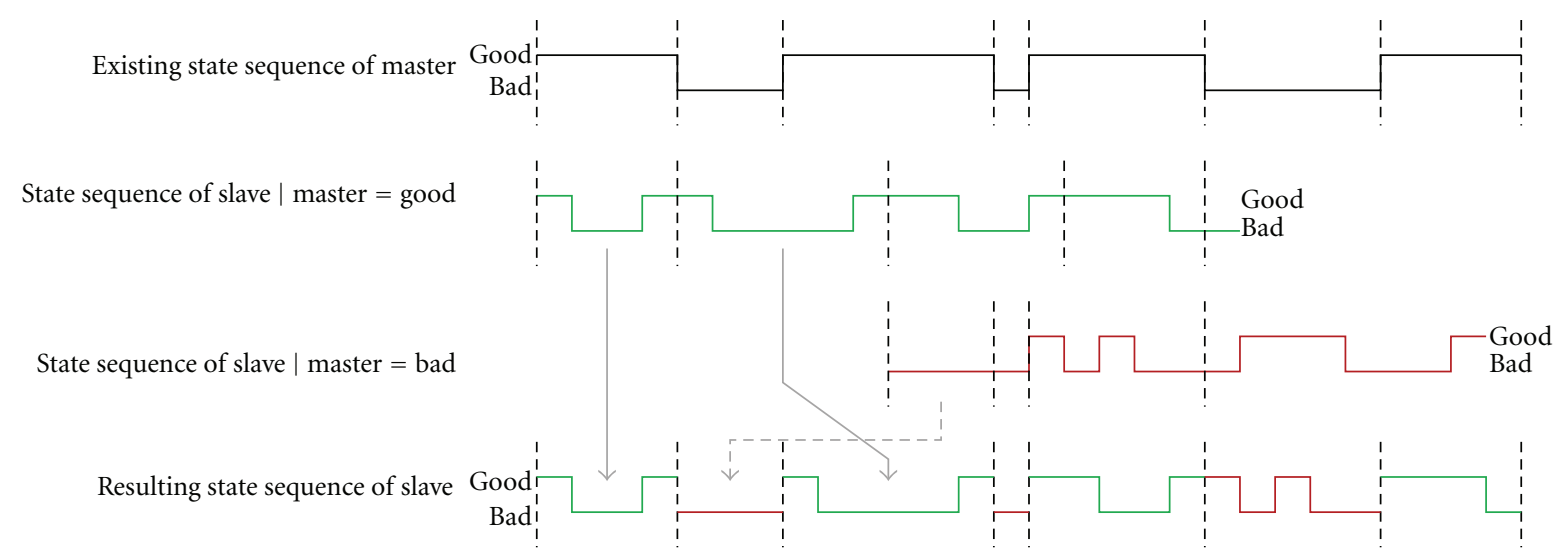

Figure 3: Realisation of Master-Slave with the Conditional Assembling Method: Given a master sequence, two independent conditional slave sequences are generated for the cases "master is good" and "master is bad." Different state models (1st-order Markov, semi-Markov, etc.) can be chosen individually therefore. Finally, the slave sequence is composed by piecewise assembling the two conditional sequences according to the master sequence. In this way the correlation coefficient between master and slave, the individual state probabilities, and the combined state probabilities can be accurately described.

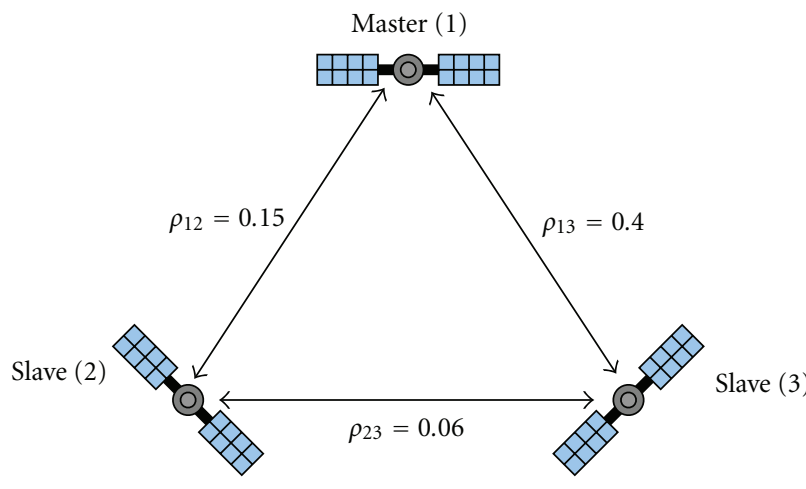

(a)

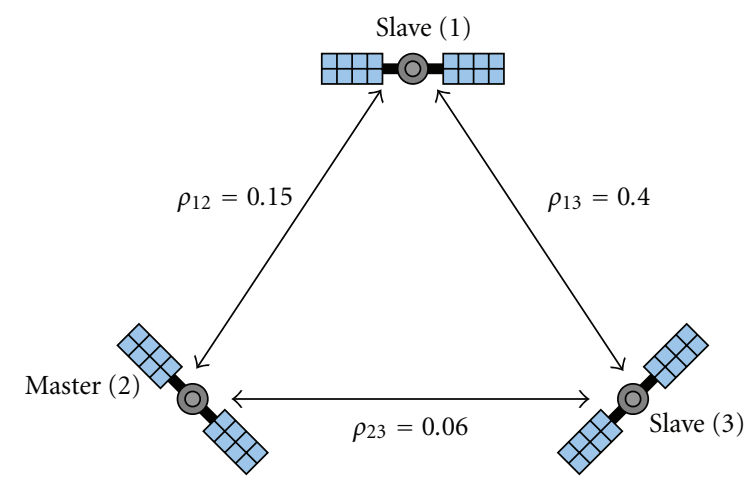

(b)

FIGURE 4: (a) Appropriate mapping of a three-satellite constellation for the Master-Slave approach (Mapping 1)—the correlation between the slaves is low. (b) Inappropriate mapping of the three-satellite constellation (Mapping 2) — the correlation between the slaves is high.

Markov model can be used. The final state sequence of the slave is composed of parts from both conditional sequences according to the current master state. To provide a sampleby-sample generation of the slave state sequence, the current conditional slave state generator is kept in "standby" mode when a state transition of the master is obtained.

In case that the chosen Markov model accurately describes the state probabilities of the master as well as the conditional state probabilities of the slave in case of a constant master state, then the state probabilities of the slave, the joint state probabilities, and the correlation coefficient between the satellites are perfectly remodelled. Furthermore, the state durations of the master sequence can be modelled with arbitrary accuracy (by, e.g., taking a complex model such as dynamic Markov).

However, the Conditional Assembling Method has some limitations in state duration modelling of the combined states ("good good," "good bad," etc.) and of the slave states (cf. Section 4.4).
3.2. Exemplary Evaluation of Master-Slave. To validate the Master-Slave concept for multisatellite reception, Figure 4 shows an exemplary constellation of three satellites including correlation coefficients as obtained during SDARS measurements from the project MiLADY [9, 10]. Since only the correlation between the master and slaves is described, the simulation results depend highly on the definition of the master satellite. For this purpose, in Figure 4 two mappings (Mapping 1 and Mapping 2) are defined by assuming different positions of master and slave satellites. Based on these two definitions, Figure 5 shows the resimulation results for the Master-Slave approach in terms of correlation coefficients, state probabilities, and mean state durations.

In case of Master-Slave the slave satellites are modelled independently. In theory, the correlation coefficient between two independent state sequences is zero. In fact, within Master-Slave the correlation coefficient between the slaves $\rho_{\text {slaves }}$ depends on the individual correlation coefficients 


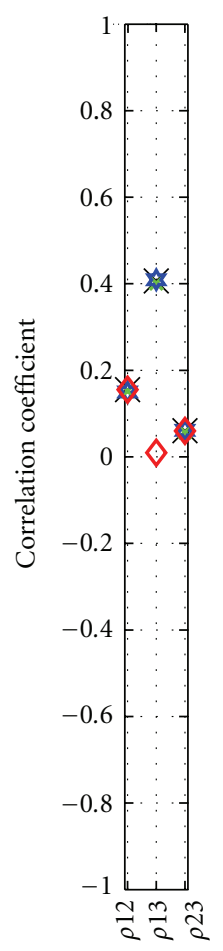

(a)

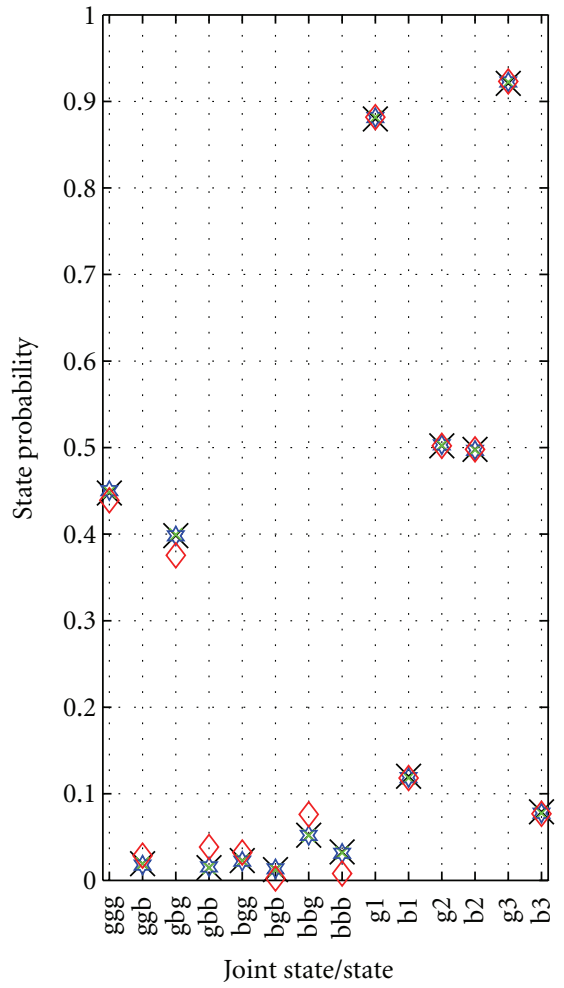

(b)

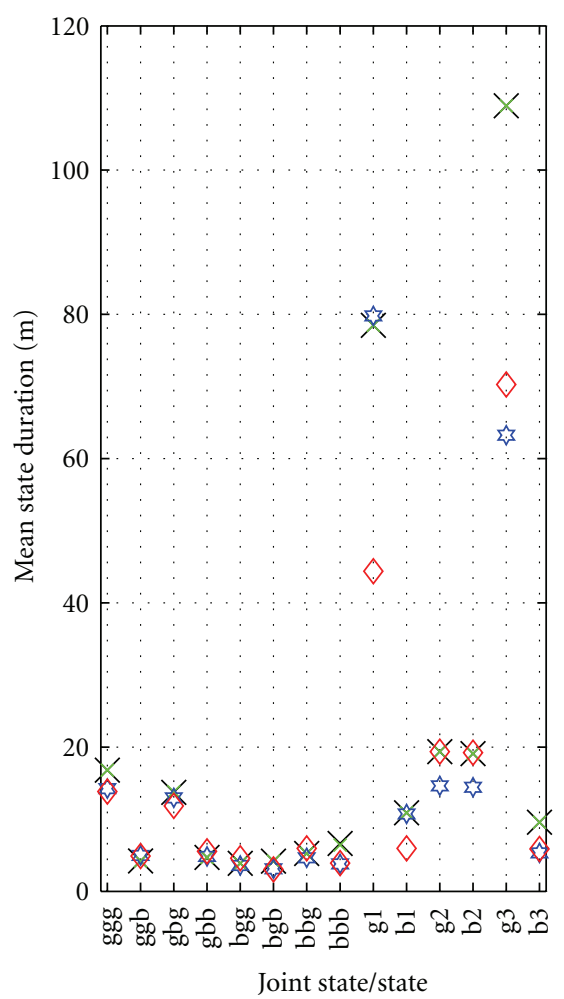

(c)

$$
\begin{array}{ll}
X \text { Measured (reference) } & \checkmark \text { Master-Slave, Mapping } 1 \\
\times \text { Markov 1st-order } & \diamond \text { Master-Slave, Mapping 2 }
\end{array}
$$

Figure 5: Correlation coefficient (a), state probability (b), and mean state duration (c) for the three-satellite constellation mappings (Mapping 1 and Mapping 2) from Figure 4. Data was taken from measurements of $5.4 \mathrm{~km}$ length in a suburban environment. Resimulation gives the following results: clearly, the resulting correlation coefficient between the slave satellites deviates for Mapping 2. The joint state probability is modelled accurately in case of Mapping 1, while Mapping 2 shows deviations. The mean state duration shows deviations in all cases (notation of states: g— "good," b—“bad," ggg—“good good good," etc.).

between master and the slaves (cf. (9) in the appendix). It always holds $\left|\rho_{\text {slaves, Master-Slave }}\right| \leq\left|\rho_{\text {slaves, measured }}\right|$.

For comparison, the results of a first-order Markov model (using a $8 \times 8$ STPM for 8 joint states) are presented. It is obtained that the first-order Markov model enables an accurate description of correlation coefficients between all satellites, of state probabilities, and of average state durations for single satellite states and combined states. The MasterSlave realisation with Conditional Assembling Method in this example is based on the first-order Markov model as well (i.e., both the master state sequence and the conditional state sequences of the slaves are modelled with first-order Markov chains). The result is that for Mapping 1 and Mapping 2 the state probabilities of the individual satellites, the correlation coefficients between master and slaves, and the mean state durations of the master (which has a different position in the examples) are modelled accurately.

Mapping 1 was defined such that the correlation between the slaves is low (with $\rho_{\text {slaves }}=0.06$ ). The higher correlation coefficients between master and slaves $\left(\rho_{12}=0.15\right.$ and $\left.\rho_{13}=0.40\right)$ provide some correlation of the slaves as well. In Mapping $1 \rho_{\text {slaves }}$ is remodelled accurately. As a consequence, also the joint state probabilities ("good good good", ..., "bad bad bad") are remodelled accurately.

In Mapping 2 the correlation between the slaves is high (with $\rho_{\text {slaves }}=0.40$ ). Due to low correlation coefficients between the master and the slaves $\left(\rho_{12}=0.15\right.$ and $\left.\rho_{23}=0.06\right), \rho_{\text {slaves }} \approx 0$ and deviates strongly from the measurements. As a result, the joint state probabilities are not accurately remodelled. For the application of system planning it should be noted that in case of describing an insufficient correlation a higher diversity gain for the multisatellite constellation will be predicted.

Hence, Mapping 2 suggests a lower probability of the combined state "bad bad bad" than obtained from measurements. Such a modelling error should be avoided. A solution for Master-Slave is an appropriate definition of master and slave satellites. Therefore, in Section 4 the performance of Master-Slave for different positions of master and slaves is evaluated. 


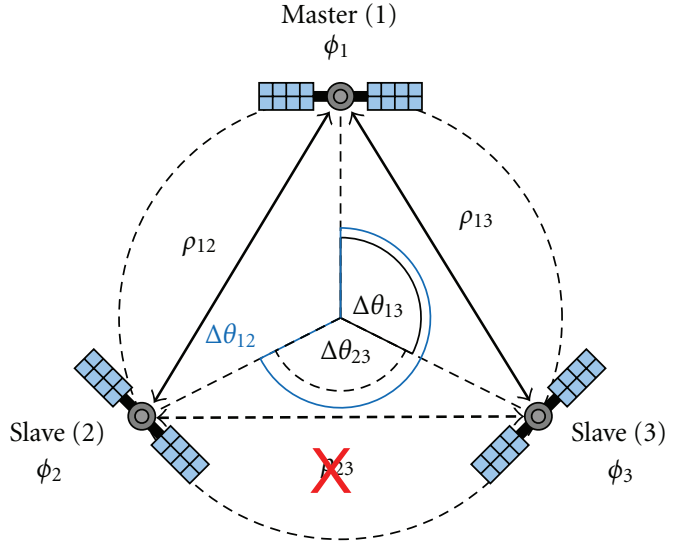

FIGURE 6: Three-satellite constellation for the Master-Slave analysis with elevation angles $\phi$, azimuth angle separations $\Delta \theta$, and correlation coefficients $\rho$.

\section{Validation of State Probability Modelling with Master-Slave}

This section will focus on the modelling accuracy when using the Master-Slave approach incorporating three satellites. Quality criterion is the deviation of the joint "bad" state probability $\left(\Delta P_{\mathrm{bbb}}\right)$, depending on the azimuth and elevation constellation. At the end of this section, we will derive constraints for appropriate satellite constellations and how to map them into the Master-Slave context.

4.1. Analysis of a Three-Satellite System. Basis of the analysis is a three-satellite constellation according to Figure 6. In the sequel, the master satellite is always referred to as index 1 , while indices 2 and 3 refer to the slaves. The constellation (and the mapping) is described by the elevation angles $\phi_{1}$, $\phi_{2}$, and $\phi_{3}$ and the azimuth angle separations $\Delta \theta_{12}, \Delta \theta_{13}$, and $\Delta \theta_{23}$. Following the Master-Slave approach, only the correlations between master and slave are described $\left(\rho_{12}\right.$ and $\left.\rho_{13}\right)$, while the correlation between the slaves $\left(\rho_{23}\right)$ is neglected. In the following, the consequences of the neglected correlation $\rho_{23}$ are investigated.

4.1.1. Influence of the Azimuth Angle Separation. Figure 7 shows the state-correlation coefficient $\rho$ between two satellites with dependency on their azimuth angle separation $\Delta \theta$. The elevation angles of both satellites are $\phi_{1}=\phi_{2}=15^{\circ}$; the driving direction can be assumed as uniformly distributed between 0 and $360^{\circ}$. These data were derived from analysing dual-satellite constellations of GNSS measurements in urban environments during the project MiLADY and are presented in detail in [7]. It can be observed that low azimuth angle separations lead to a high correlation, while for $\Delta \theta=$ $45^{\circ} \cdots 150^{\circ}$ the satellites are almost uncorrelated. A slight increase of the correlation is obtained for the antipodal constellation $\left(\Delta \theta \approx 180^{\circ}\right)$. Due to the uniformly distributed driving direction the graph is symmetrical to $\Delta \theta=0^{\circ}$ and $\Delta \theta=180^{\circ}$.

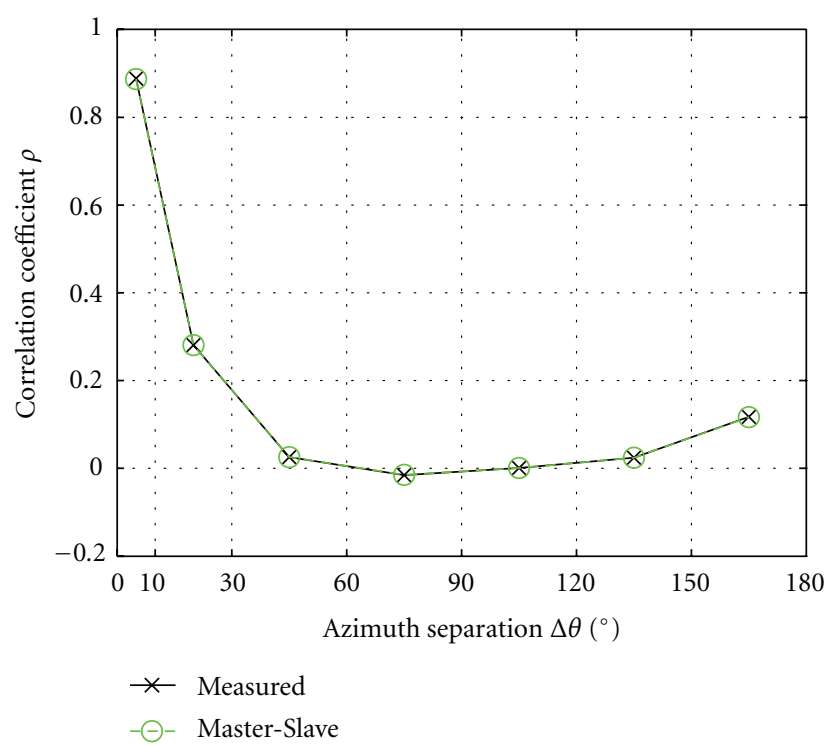

Figure 7: State correlation coefficient $\rho$ between two satellites with dependency on the azimuth angle separation $\Delta \theta$ derived from measurements and recalculated with Master-Slave. The MasterSlave approach with Conditional Assembling Method presented in this paper resimulates accurately the correlation coefficients for the two-satellite constellation (master and slave). The elevation angles are $\phi_{1}=\phi_{2}=15^{\circ}$.

Based on these results for the dual-satellite case we now analyse the consequences of neglecting the slave correlation $\rho_{23}$ in the Master-Slave approach. Following the configuration according to Figure 6 , we compare $\rho_{23}$ after resimulation using the Master-Slave approach versus the measured results. Figure 8 shows both cases with dependency on each of the azimuth angle separations $\Delta \theta_{12}, \Delta \theta_{13}$, and $\Delta \theta_{23}$. The error is given by $\Delta \rho_{23}=\mid \rho_{23}$, measured $-\rho_{23}$, Master-Slave $\mid$. Clearly, this error is large when the two slaves come close to each other, that is, $\Delta \theta_{12} \approx \Delta \theta_{13}$ - except when one slave is close to the master.

Besides correctly remodelling the state correlation, for designing mobile satellite communication systems a very important parameter is the probability of all satellites being in "bad" state, that is, $P_{\mathrm{bbb}}$. It characterises the probability of service unavailability for the entire system and requires special attention, therefore. According to Figure 9, in the measured scenario $P_{\text {bbb }} \approx 0.8$ if all three satellites are colocated, and $P_{\mathrm{bbb}} \approx 0.6$ if two of the three satellites are colocated. Considering the probability error $\Delta P_{\text {bbb }}=\mid P_{\text {bbb, measured }}-$ $P_{\text {bbb, Master-Slave }}$ (the dashed red line in Figure 9) as a quality measure of the modelling approach, we can extend the analysis to further parameters. Therefore, in the next section the elevation-angle dependency is investigated.

For the sake of completeness, we show the consequences when neglecting not only the correlation between the slaves, but neglecting a further correlation as well as neglecting all correlations in the three-satellite system: as can be observed from Figure 10, for azimuth angle separations of $\Delta \theta \leq \approx 30^{\circ}$ 

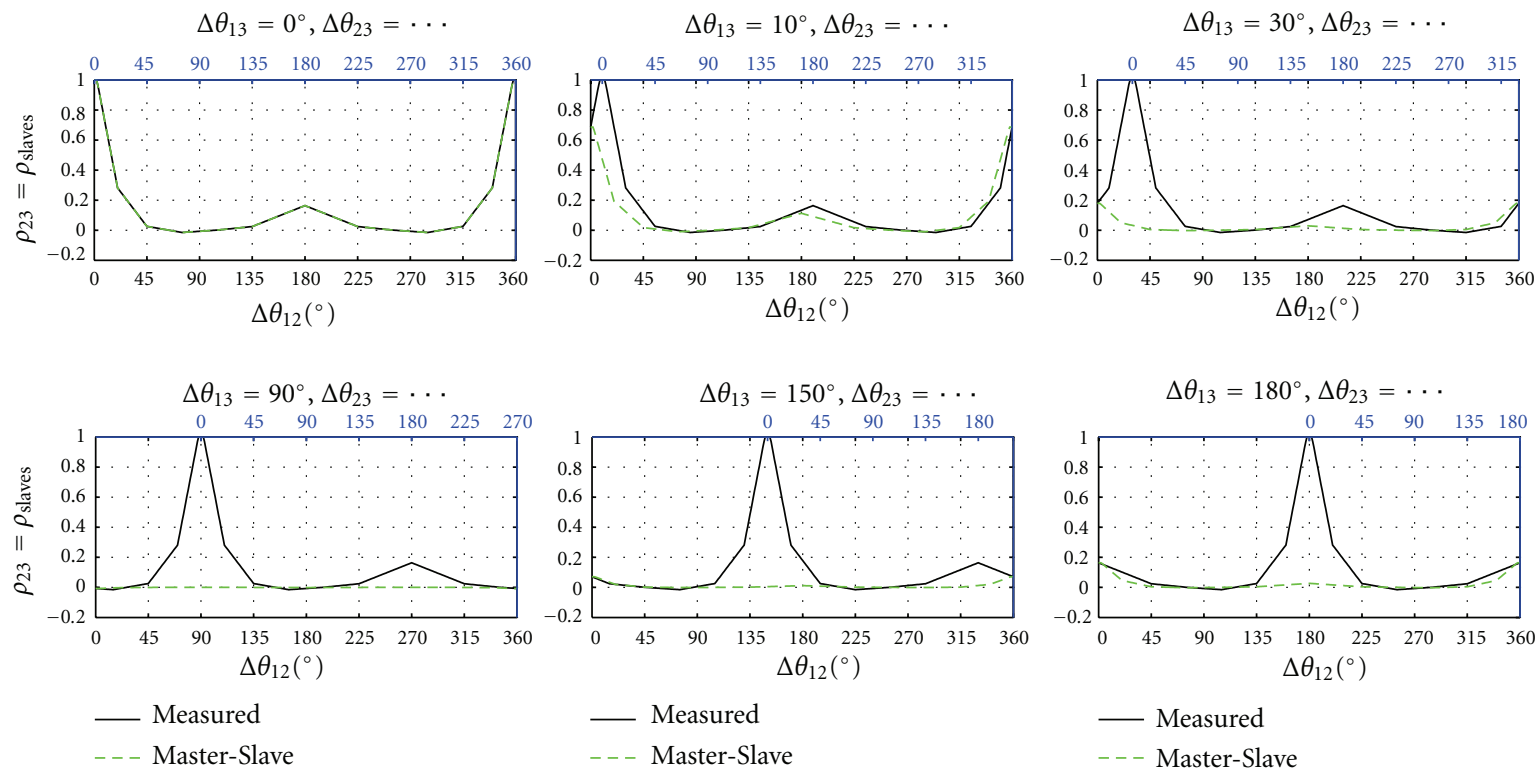

- - - Master-Slave

FIgURE 8: Correlation coefficients between the slaves $\left(\rho_{23}\right)$ with dependency on the azimuth angle separations $\Delta \theta_{12}, \Delta \theta_{13}$, and $\Delta \theta_{23}$ from measurements and recalculated with Master-Slave. $\Delta \theta_{13}$ is kept constant in each subfigure. The elevation angles are $\phi_{1}=\phi_{2}=\phi_{3}=15^{\circ}$.
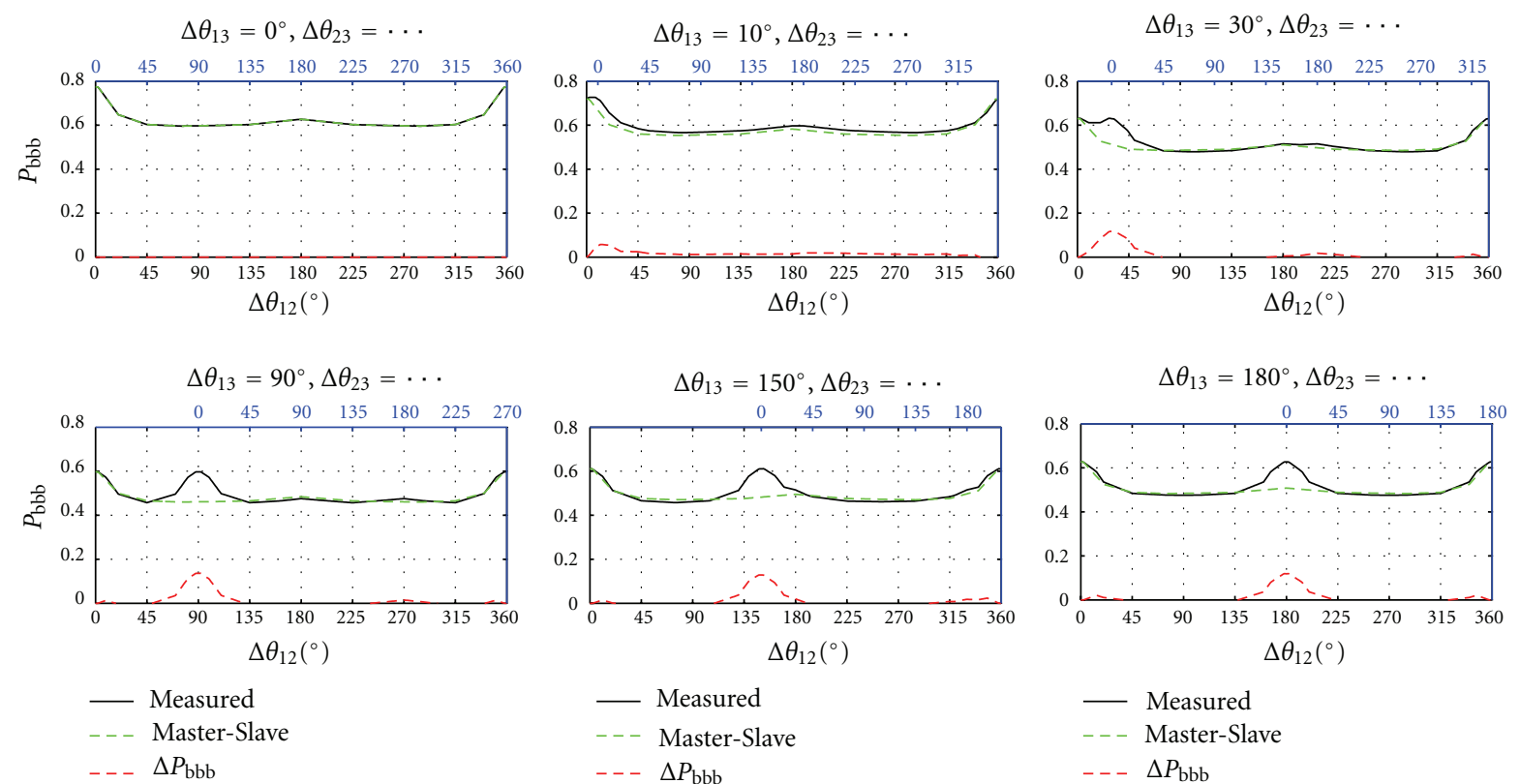

FIGURE 9: "bad bad bad" state probabilities $\left(P_{\mathrm{bbb}}\right)$ of three satellites for different azimuth angle separations $\Delta \theta$ from measurements and recalculated with Master-Slave. The elevation angles are $\phi_{1}=\phi_{2}=\phi_{3}=15^{\circ}$, where the "bad" state probability of each individual satellite is $P_{\mathrm{b}}=0.77$.

the correlation cannot be neglected. Furthermore, the correlation should be considered for antipodal constellations, as

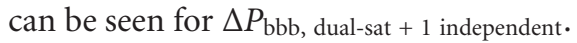

4.1.2. Influence of the Elevation Angles. Based on the validation of Master-Slave with dependency on the azimuth angle separation, we now incorporate the elevation angles as further variables. The Figures 11 and 12 show the probability error $\Delta P_{\mathrm{bbb}}$ with Master-Slave with dependency on the azimuth separations $\Delta \theta_{12}$ and $\Delta \theta_{13}$ for different combinations of elevation angles between master, slave satellite 2, and slave satellite 3 . Figure 11 shows results with a constant master elevation $\phi_{1}=15^{\circ}$, Figure 12 for $\phi_{1}=45^{\circ}$, respectively. The figures include also the results from Figure 10 for the special case $\phi_{1}=\phi_{2}=\phi_{3}=$ $15^{\circ}$. Since the slave satellite 2 , and slave satellite 3 can be exchanged, only the lower triangle is shown. From Figures 11 and 12 appropriate and inappropriate constellations (and 


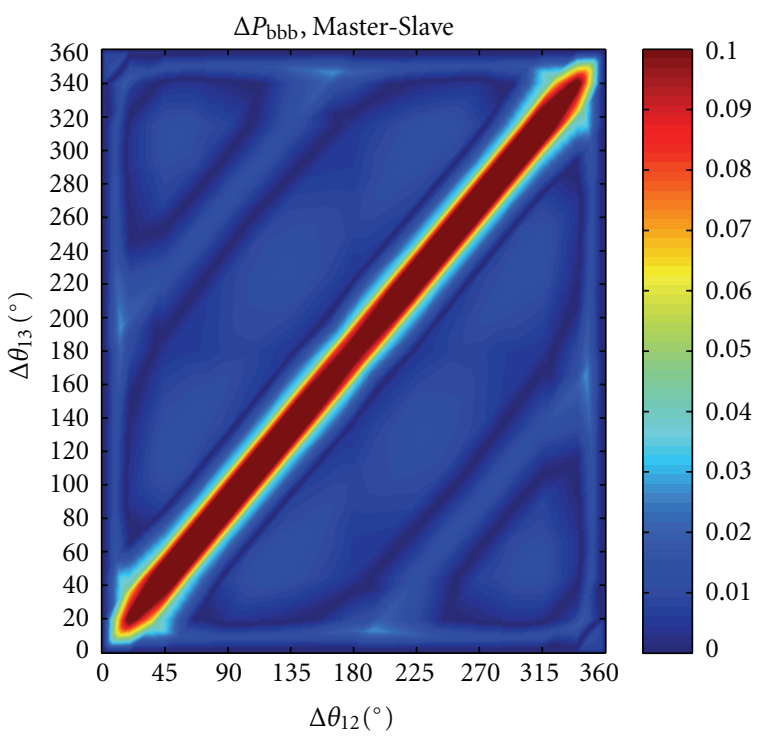

(a)

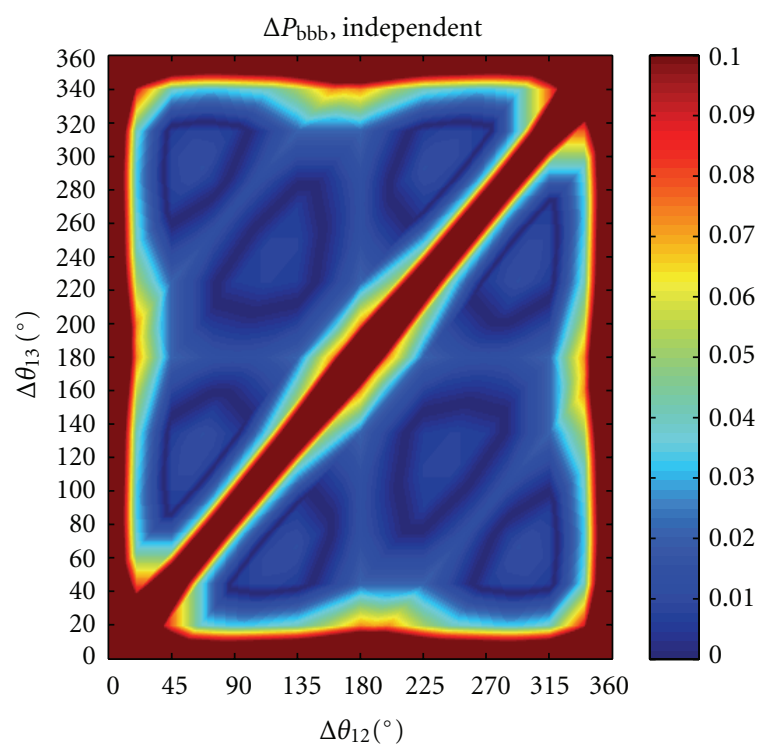

(b)

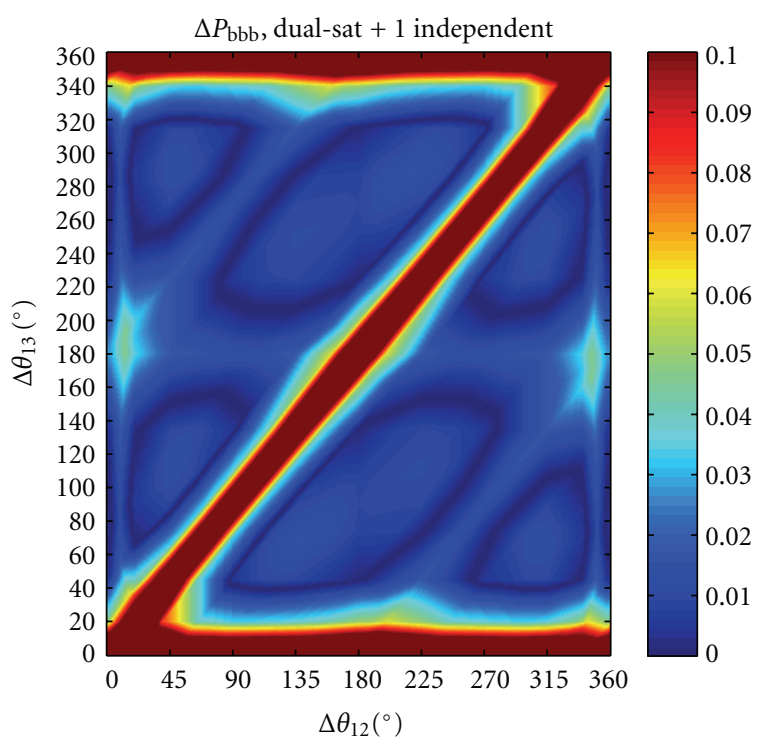

(c)

Figure 10: Performance comparison for three-satellite modelling between Master-Slave method (a), independent modelling (b), and a twosatellite model + one independent satellite (c). The figures show the difference between measured and resimulated "bad bad bad" state probability $\left(\Delta P_{\mathrm{bbb}}\right)$ for different azimuth angle separations $\left(\theta_{12}\right.$ and $\left.\theta_{13}\right)$ of two slave satellites. The elevation angles are $\phi_{1}=\phi_{2}=\phi_{3}=15^{\circ}$.

mappings) for the three-satellite Master-Slave approach can be determined. The following is obtained.

(i) Assuming constant elevation angles (represented by one subfigure), the error $\Delta P_{\text {bbb }}$ is maximal for a low azimuth separation between the slaves, while some azimuth separation to the master exists. This error can be avoided by redefinition of the system such that the low azimuth separation is between the master and one of the slaves.

(ii) If one of the slaves has a higher elevation than the master and the second slave (e.g., $\phi_{1}>\phi_{2}$ and $\phi_{3}$ ), then the error $\Delta P_{\mathrm{bbb}}$ for low slave separations is reduced with respect to $\phi_{1}=\phi_{2}=\phi_{3}$. The reason is that the correlation coefficient and therefore the correlation error between slaves are lower due to the elevation angle separation.

(iii) The error $\Delta P_{\mathrm{bbb}}$ with Master-Slave is high when both slaves have a higher elevation than the master.

(iv) Comparing Figures 11 and 12, it is obtained that a master with a high elevation has great benefits. For all positions of the slaves the error $\Delta P_{\mathrm{bbb}}$ for elevation $\phi_{1}=45^{\circ}$ is much lower than for $\phi_{1}=15^{\circ}$.

(v) Comparing the constellations $\phi_{1}=\phi_{2}=\phi_{3}=$ $15^{\circ}$ and $\phi_{1}=\phi_{2}=\phi_{3}=45^{\circ}$ it is seen that 

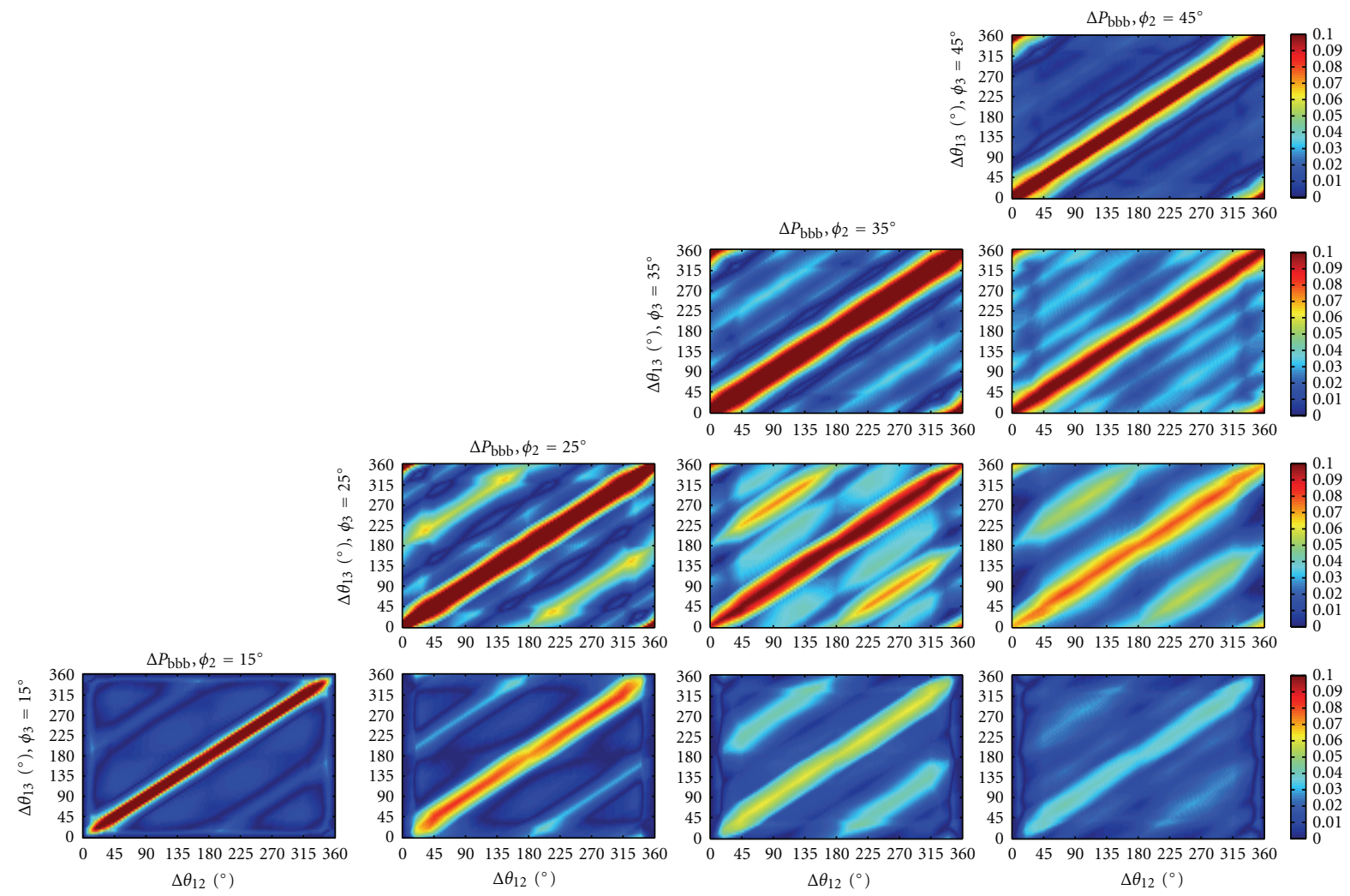

FIGURE 11: Difference between measured and resimulated "bad bad bad" state probability $\left(\Delta P_{\mathrm{bbb}}\right)$ for different elevation angles $\left(\phi_{2}\right.$ and $\left.\phi_{3}\right)$ and azimuth angle separations $\left(\theta_{12}\right.$ and $\left.\theta_{13}\right)$ of two slave satellites. The master elevation is constant at $\phi_{1}=15^{\circ}$.

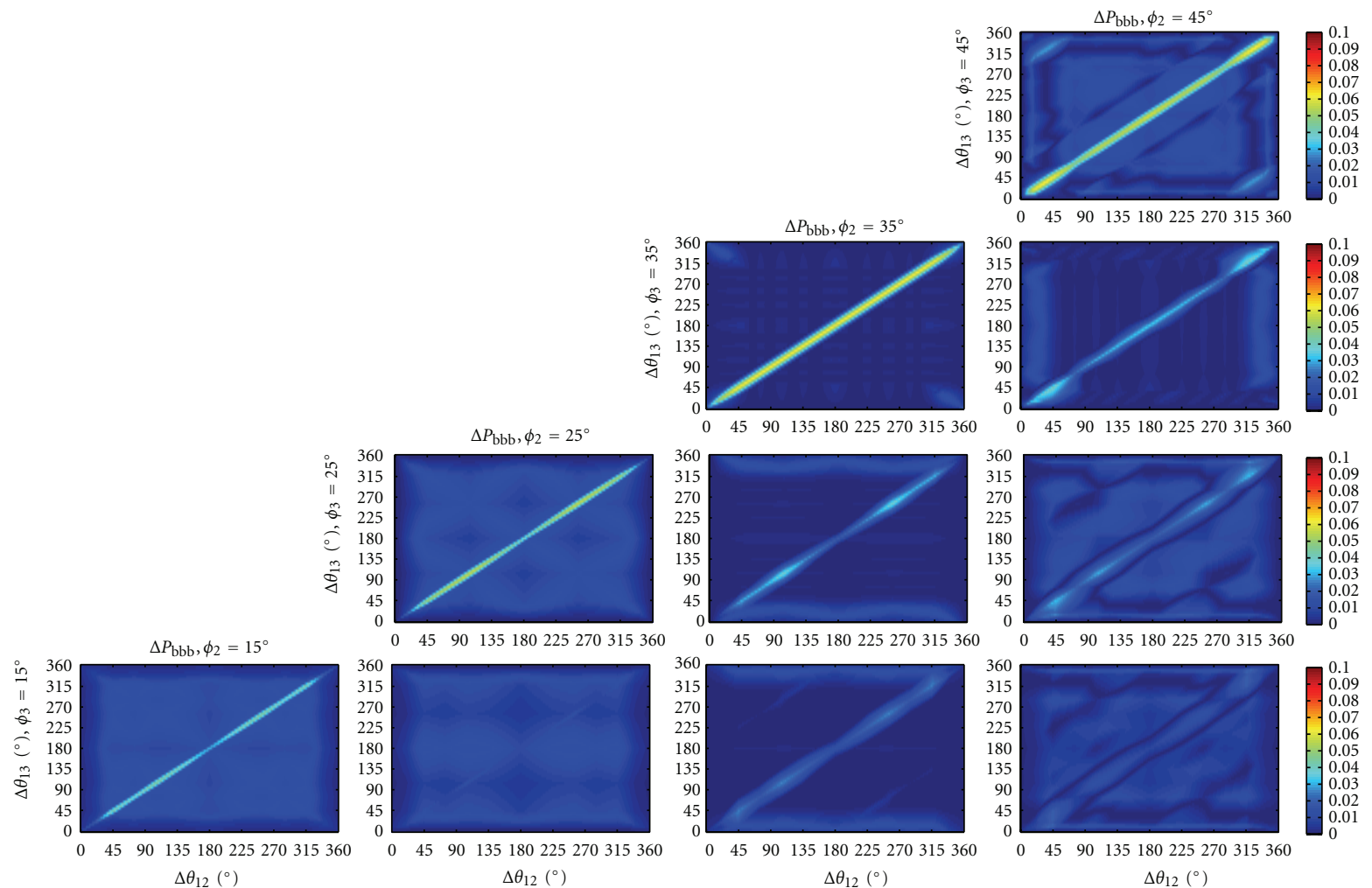

FIGURE 12: Difference between measured and resimulated "bad bad bad" state probability $\left(\Delta P_{\mathrm{bbb}}\right)$ for different elevation angles $\left(\phi_{2}\right.$ and $\left.\phi_{3}\right)$ and azimuth angle separations $\left(\theta_{12}\right.$ and $\left.\theta_{13}\right)$ of two slave satellites. The master elevation is constant at $\phi_{1}=45^{\circ}$. 

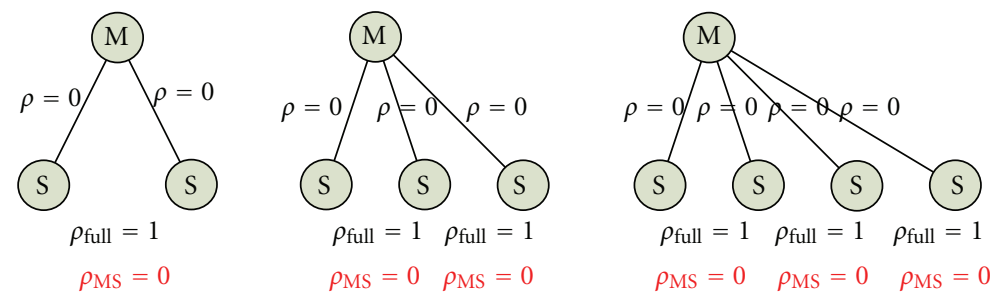

FIGURE 13: Worst cases for Master-Slave modelling: there is no correlation between master and slave satellites $(\rho=0)$, but full correlation between the slaves $\left(\rho_{\text {slaves }}=1\right)$.

the higher elevation angles provide a lower probability error $\Delta P_{\mathrm{bbb}}$. It should be noted that the correlation error $\Delta \rho$ in case of some elevation angles is similar. This result is therefore based on the satellite state probabilities, which depends on the current elevation angle. An analysis of the relation between single satellite state probabilities and the probability error with Master-Slave is found in Section 4.2.

(vi) A great performance difference is seen between the constellations $\phi_{1}=15^{\circ}, \phi_{2}=45^{\circ}$, and $\phi_{3}=45^{\circ}$ and $\phi_{1}=45^{\circ}, \phi_{2}=45^{\circ}$, and $\phi_{3}=15^{\circ}$. Although the satellites have the same position on the hemisphere, for the second mapping nearly no limitations of azimuth angle separations are obtained. An examination on the influence of different elevation angles of master and slave satellites is done in Section 4.2.

4.2. Estimation of Worst-Case Probability Error for MasterSlave with Three, Four, and Five Satellites. From the results in the previous section it is known that a high probability error occurs when master and slave satellites are weakly correlated, but the correlation between slaves is high. Worst case would be no correlation between master and slave and full correlation between slaves. Figure 13 shows worst cases for Master-Slave with three, four, and five satellites (please note that $\rho=1$ between the satellites is not realistic, since different satellites must be exactly colocated therefore) Based on these worst-case constellations, an examination on the probability error $\Delta P_{\text {all bad }}$ (we define $P_{\text {all bad }}$ as the worst reception case of the multisatellite system. It includes $P_{\mathrm{bb}}, P_{\mathrm{bbb}}$, and $P_{\mathrm{bbbb}}$ for a system with two, three, and four satellites, respectively) for the Master-Slave approach with more than three satellites is possible.

The highest satellite diversity gain $\left(=P_{\mathrm{b}}-P_{\text {all bad }}\right)$ with a multisatellite system is provided if all satellites are uncorrelated $(\rho=0)$. In this case, the probability of the combined state is the product of the individual state probabilities: $P_{\mathrm{allbad}}=P_{\mathrm{b}}^{k}$ with $k$ being the number of satellites. In contrast, a multisatellite system has no gain if all satellites are fully correlated $(\rho=1)$. Then it holds $P_{\text {all bad }}=$ $P_{\mathrm{b}}$.

Assuming the constellations in Figure 13 the MasterSlave model would provide uncorrelated slave satellites with $\rho=0$. As a consequence, the Master-Slave model has $k$ uncorrelated channels and for the combined state results $P_{\text {all bad }}=P_{\mathrm{b}}^{k}$ (assuming same state probabilities of the individual satellites). In fact, the multisatellite systems in Figure 13 have only two uncorrelated channels. The probability error of Master-Slave is therefore $\Delta P_{\text {all bad }}=P_{\mathrm{b}}^{2}-P_{\mathrm{b}}^{k}$.

Figure 14 shows the worst-case probability error $\Delta P_{\text {all bad }}$ with dependency on the "bad" state probability $P_{\mathrm{b}}$ of the individual satellites. It represents that the reception for all satellites have the same elevation. As a general tendency, a reduction of the probability $P_{\mathrm{b}}$ (e.g., due to an increased elevation) of the single satellites provides a reduction of $\Delta P_{\text {all bad }}$ This situation was obtained in Figures 11 and 12 between constellations $\phi_{1}=\phi_{2}=\phi_{3}=15^{\circ}$ and $\phi_{1}=\phi_{2}=$ $\phi_{3}=45^{\circ}$.

Figure 15 evaluates the worst-case probability error $\Delta P_{\text {all bad }}$ for different elevation angles of the master and the slaves. It is seen that a high elevation angle of the master provides a lower error $\Delta P_{\text {all bad }}$.

4.3. Appropriate Constellations for Master-Slave Approach with Three, Four, and Five Satellites. This section concludes appropriate and inappropriate satellite positions for MasterSlave with three satellites and extends it for the multisatellite case.

Figure 16 (left) shows combinations of azimuth separations between master (1) and slave (2) $\Delta \theta_{12}$ and master (1) and slave (3) $\Delta \theta_{13}$ for which the modelling error $\Delta P_{\text {bbb }}$ exceeds a certain threshold. Assuming that $\Delta P_{\text {bbb }} \leq 0.05$ is an acceptable modelling result, appropriate and inappropriate regions (in terms of $\Delta \theta$ ) are found therefore for the MasterSlave approach with three satellites.

Concrete examples are given in Figure 16: by defining a certain position of satellite 2 (it has initially no constraints in terms of appropriate positions) the inappropriate azimuth separations for satellite 3 are detected. Figure 17 presents appropriate zones for satellite 3 for predefined positions of satellite 2 in a polar diagram. Given the example in Figure 16, the inappropriate positions of satellite 3 are $\Delta \theta_{13}=\Delta \theta_{12} \pm$ $20^{\circ}$ - except when satellite 2 is close to the master.

Outgoing from the three-satellite analysis, a quantitative estimation for inappropriate zones for systems with more than three satellites is possible. It is done by decomposing the multisatellite constellation into combinations of each one master and two slaves. For example, from a four-satellite system the combinations master-slave2-slave3, master-slave2slave4, and master-slave3-slave4 are separately evaluated. The result is that inappropriate positions of each slave depend on the position of another slave. Based on this method, 


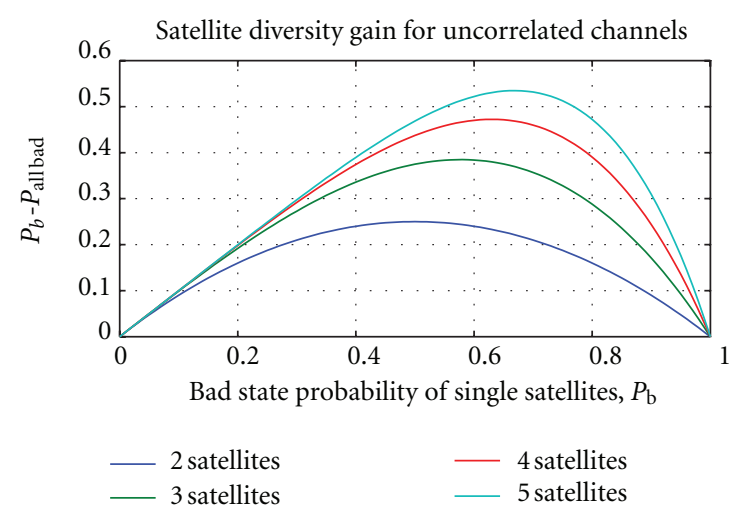

(a)

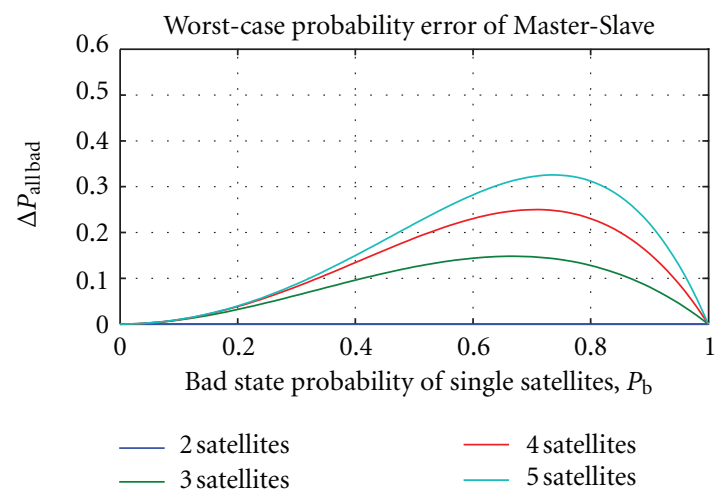

(b)

Figure 14: (a) Satellite diversity gain of uncorrelated satellites with dependency on the bad state probability of the individual satellites $P_{\mathrm{b}}$, and the number of satellites $k$. It represents the improvement of a system with $k$ uncorrelated channels to a system with one channel. (b) Worst-case probability error of the Master-Slave approach (constellations from Figure 13) with dependency on the bad state probability of the individual satellites, and the number of satellites (it is $k$ uncorrelated channels minus 2 uncorrelated channels).

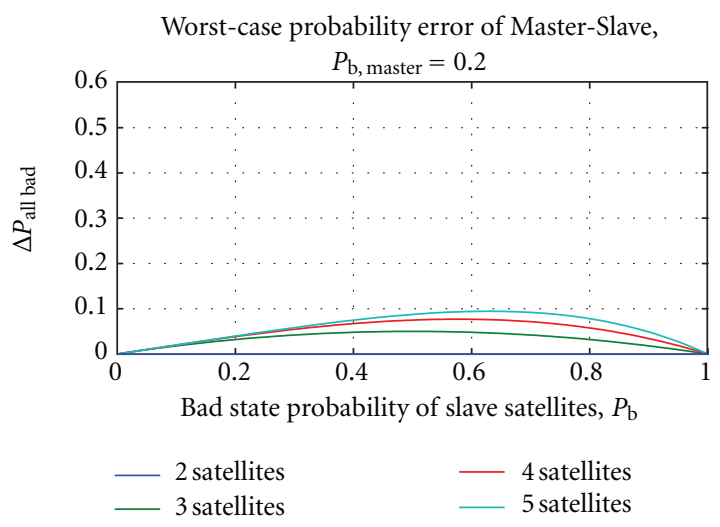

(a)

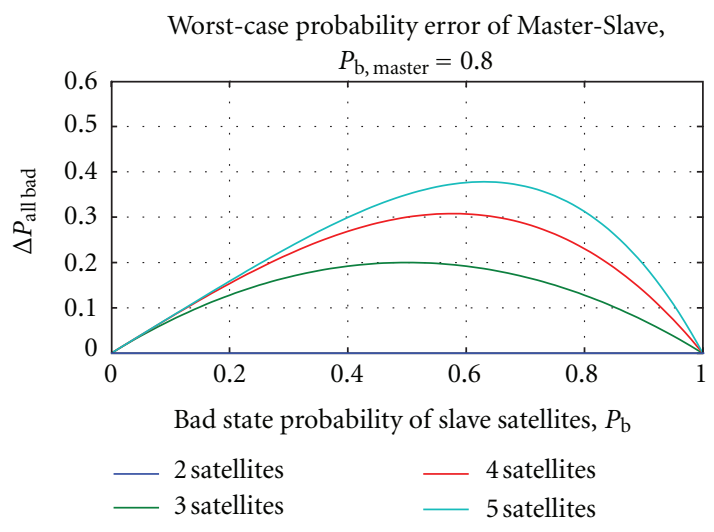

(b)

Figure 15: Worst-case probability error of the Master-Slave approach (constellations from Figure 13) with dependency on the bad state probability of the slave satellites, the number of satellites, and the master probability $P_{\mathrm{b}, \text { Master }}=0.2(\mathrm{a})$ and $P_{\mathrm{b}, \text { Master }}=0.8$ (b). A low bad state probability of the master ( $\hat{=}$ high elevation) is beneficial.

Figure 18 shows exemplary constellations of a four-satellite system with predefined positions of satellites 2 and 3. The result is possible positions of satellite 4, for which the Master-Slave approach provides reliable results. In one of the constellations (third from left) there is no option for satellite 4, since the constellation between S2 and S3 is already inappropriate.

Figure 19 shows constellations of a system with five satellites. Appropriate positions of satellite 5 are resulting from defined positions of satellites 2,3 , and 4 .

It can be concluded that with a higher number of satellites the range of appropriate positions decreases.

Please remember that the "inappropriate zones" define only constellations where $\Delta P_{\text {bbb }}$ is below a certain threshold ( 0.05 was selected exemplarily). For a system with more than three satellites it means that $\Delta P_{\text {all bad }}$ may not be simulated correctly with Master-Slave. It makes no statement about the absolute error $\Delta P_{\text {all bad. }}$. Constellations are even possible where $\Delta P_{\text {all bad }}$ can be neglected, although the Master-Slave constellation was found as inappropriate.
4.4. State Duration Modelling with Master-Slave. In Section 3.1 we presented the Conditional Assembling Method as a first realisation of the Master-Slave approach. It provides an accurate modelling of the state probabilities of the single satellites as well as of the combined satellites for dual-satellite reception. However, for the configuration of satellite broadcasting systems with long time interleaving, an accurate modelling of the fading signal over time is crucial.

Figure 20 shows therefore the state duration statistics of the "bad" state from satellite 1 and satellite 2 and of the combined "bad bad" state. The complementary state duration CDFs from measurements are compared with resimulation results of a Master-Slave model and with a dualsatellite semi-Markov model as presented in [7].

It is seen that the Conditional Assembling Method has some limitations for remodelling the duration statistic for the combined "bad bad" state and of the slave satellites' "bad" state. In contrast, the duration statistic of the master satellite can be exactly remodelled by using complex approaches like dynamic Markov chains. 

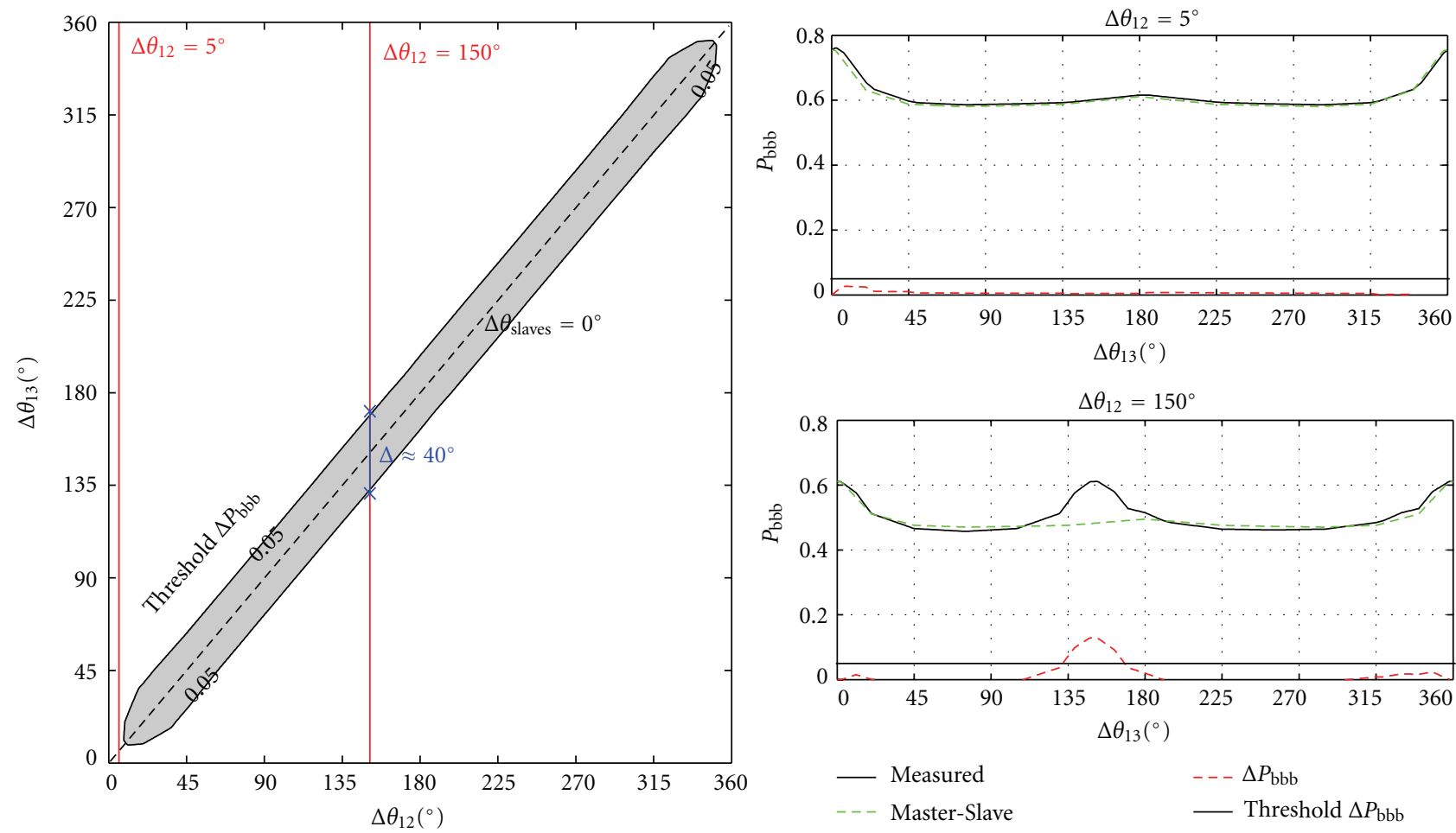

Figure 16: Left: Inappropriate Master-Slave constellations (grey field) with dependency on the azimuth angle separations between master and slaves $\left(\Delta \theta_{12}\right.$ and $\left.\Delta \theta_{13}\right)$ for $\phi_{1}=\phi_{2}=\phi_{3}=15^{\circ}$. They are assumed for $\Delta P_{\mathrm{bbb}}>0.05$ (cf. Figure 11). Right: Assuming satellite 2 has position $\Delta \theta_{12}=5^{\circ}$, then there is no constraint for the position of satellite $3\left(\Delta \theta_{13}\right)$. Assuming satellite 2 has position $\Delta \theta_{12}=150^{\circ}$, then satellite 3 with $130^{\circ} \leq \Delta \theta_{13} \leq 170^{\circ}$ is inappropriate for Master-Slave.
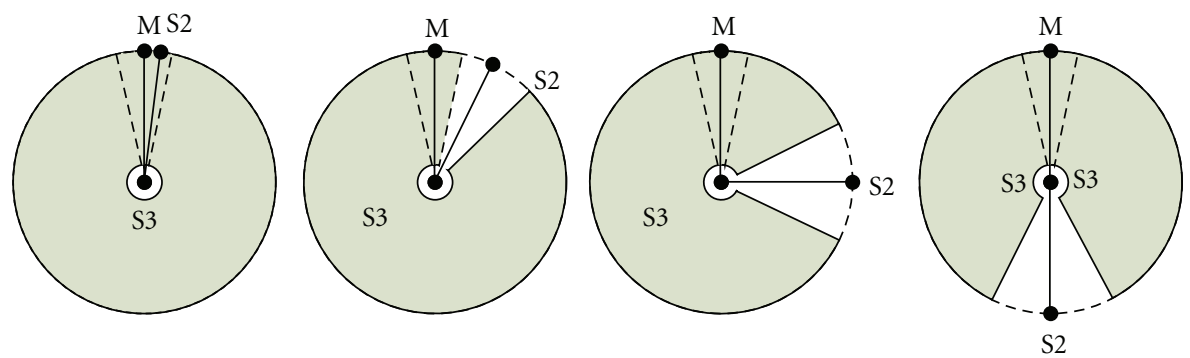

Figure 17: Appropriate constellations of three satellites for the usage of a Master-Slave approach $\left(\mathrm{M}-\mathrm{Master}, \mathrm{S} 2, \mathrm{~S} 3-\mathrm{Slaves}\right.$, with $\phi_{\mathrm{M}}=$ $\left.\phi_{2}=\phi_{3}\right)$. The coloured fields indicate appropriate positions of S3. The inappropriate positions for $\mathrm{S} 3$ are $\pm 20^{\circ}$ around $\Delta \theta_{12}$.
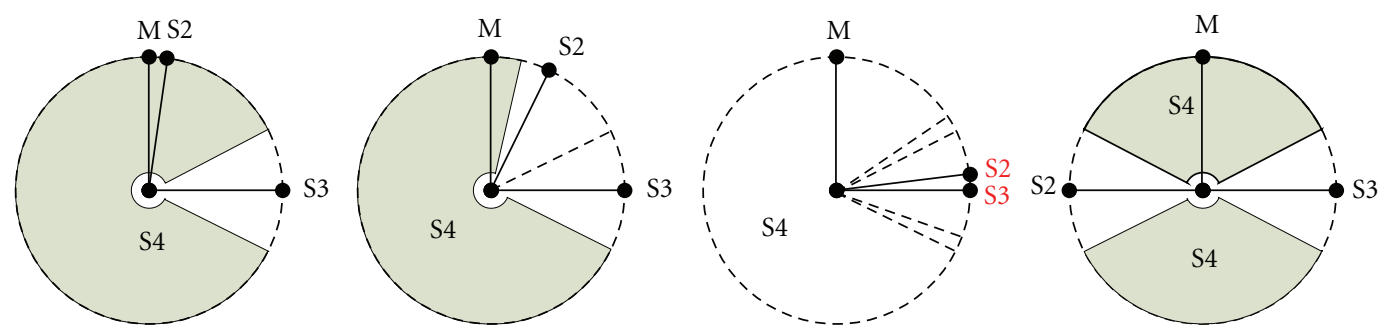

FIGURE 18: Appropriate constellations of four satellites for the usage of a Master-Slave approach $\left(\mathrm{M}-\mathrm{Master}, \mathrm{S} 2,3,4-\mathrm{Slaves}\right.$, with $\phi_{\mathrm{Master}}=$ $\left.\phi_{\text {slaves }}\right)$. The coloured fields indicate appropriate positions of S4. In the third case there is an inappropriate constellation for Master-Slave, since S2 and S3 are highly correlated. 

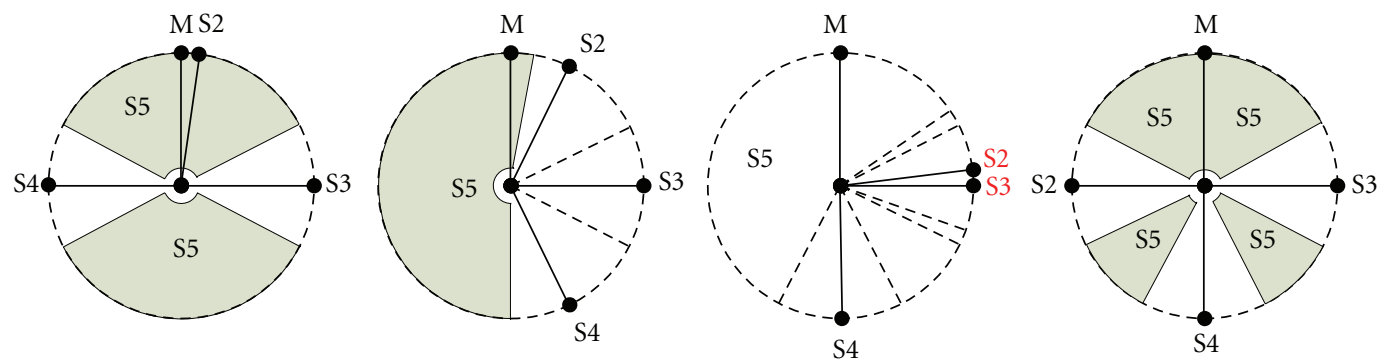

FIGURE 19: Appropriate constellations of five satellites for the usage of a Master-Slave approach. $\left(\mathrm{M}-\mathrm{Master}, \mathrm{S} 2,3,4,5-\mathrm{Slaves}\right.$, with $\phi_{\text {Master }}=$ $\left.\phi_{\text {slaves }}\right)$. The coloured fields indicate appropriate positions of S5. In the third case there is an inappropriate constellation for Master-Slave, since S2 and S3 are highly correlated.

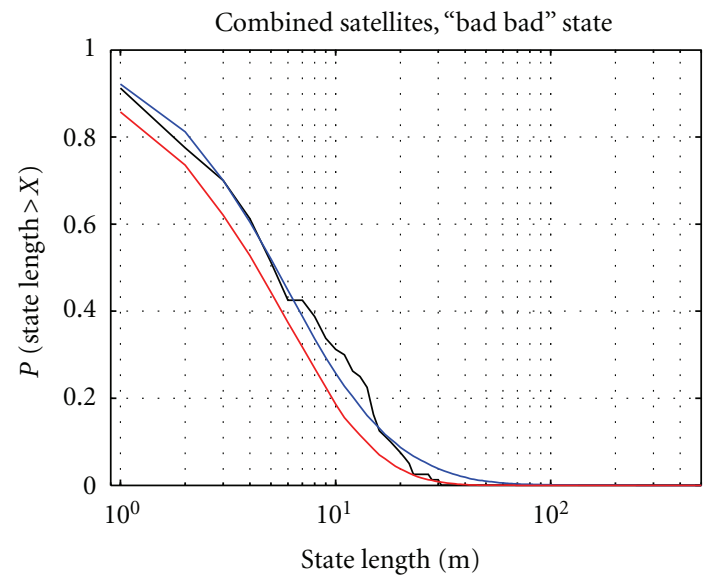

(a)

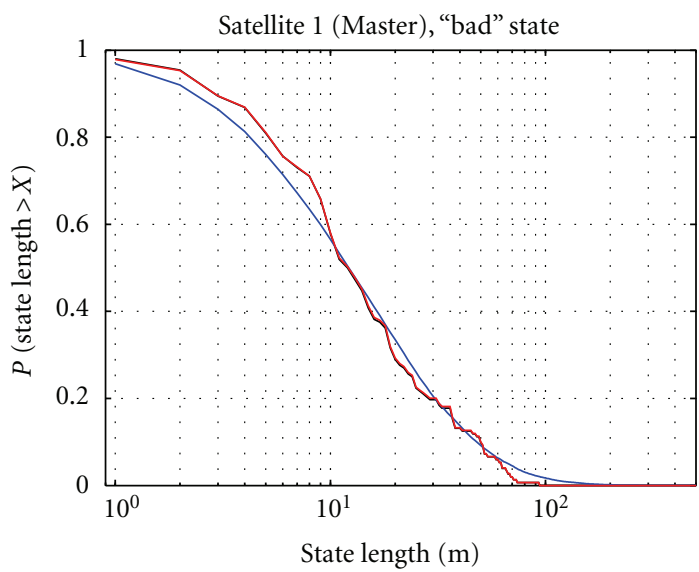

(b)

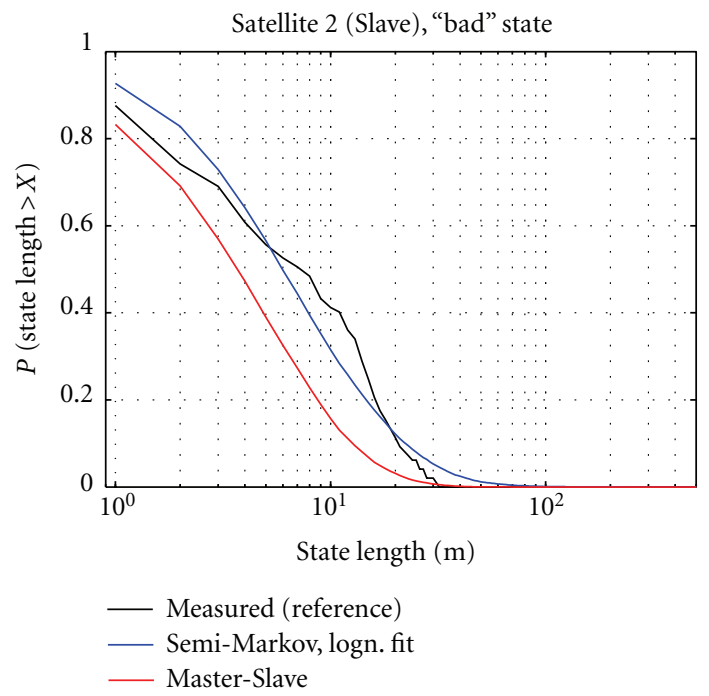

(c)

FIGURE 20: State duration statistics of the combined "bad bad" state (a) and single satellite "bad" states (b and c) derived from the measurements and resimulated with two modelling approaches. The 4-state semi-Markov approach resimulates accurately the state duration statistics. The Master-Slave approach with the "Conditional Assembling Method" resimulates accurately the state durations of the Master satellite but has limitations in duration modelling of the joint states and the slave states. 
For comparison, the dual-satellite semi-Markov model with lognormal approximation of the state duration distribution resimulates more accurately the statistics of the slave satellite and of the combined system.

It can be concluded that for state modelling of a dualsatellite system the Master-Slave approach with "Conditional Assembling Method" would not be the first choice.

An extensive validation of different Master-Slave realisations with focus on accurate state duration modelling is therefore the topic of ongoing work.

\section{Conclusions}

In this paper we presented a new approach for multisatellite state modelling: the Master-Slave approach. The primary concept is that slave satellites are modelled according to an existing master state sequence, whereas the correlation between multiple slaves is omitted. Master-Slave is therefore a generic name for a modelling concept, for which different state models can be applied (such as first-order Markov, semi-Markov, and dynamic Markov models).

As a possible realisation of Master-Slave we described the "Conditional Assembling Method". It enables an exact resimulation of the correlation coefficient, the single satellite state probabilities, and the combined state probabilities between one master and one slave.

Based on this result, a detailed performance evaluation of Master-Slave for a three-satellite system is carried out in terms of state probability modelling. Therefore, measured correlation coefficients between the satellites are compared with recalculated correlation coefficients from Master-Slave with dependency on the elevation angles and the azimuth angle separations of the three-satellite system. Afterwards, the probability of the state "bad bad bad" $\left(P_{\mathrm{bbb}}\right)$ resulting from Master-Slave is compared with analytically estimated values from measurements. The difference for $P_{\mathrm{bbb}}$ from Master-Slave and from measurements is defined as probability error. It was obtained that Master-Slave has a high probability error in case of a low azimuth separation and therefore a high correlation between the slave satellites. An exception is when one of the slaves has a high correlation to the master. Furthermore, a master satellite with a high elevation provides a lower probability error compared to a master with low elevation. It can be concluded that a probability error with Master-Slave can be mitigated by appropriate definition of master and slave satellites.

Based on the analysis with three satellites, a performance analysis of Master-Slave is extended for systems with more than three satellites in terms of state probability modelling. Therefore, appropriate and inappropriate constellations of satellites were estimated, and the probability errors for worstcase constellations with Master-Slave were predicted.

In the last part of this paper we indicated that the MasterSlave implementation of this paper (Conditional Assembling Method) has some limitations according to state duration modelling. An evaluation of state duration modelling with Master-Slave is the topic of ongoing research activities. It depends amongst others on the applied state model, such as semi-Markov and dynamic Markov and requires new concepts for the realisation of Master-Slave. However, the elaboration on state probability modelling in this paper is universally valid for Master-Slave.

\section{Appendix}

\section{State Probabilities and Correlation Coefficients for the Conditional Assembling Method}

The equilibrium state probabilities of the master states and of the conditional slave states are given by

$$
\begin{aligned}
\mathbf{p}^{\text {Master }} & =\left[\begin{array}{ll}
P_{\mathrm{g}}^{\mathrm{M}} & P_{\mathrm{b}}^{\mathrm{M}}
\end{array}\right]^{T}, \\
\mathbf{p}^{\text {Slave } \mid \text { Master=good }} & =\left[\begin{array}{ll}
P_{\mathrm{g} \mid \mathrm{g}}^{\mathrm{S}} & P_{\mathrm{b} \mid \mathrm{g}}^{\mathrm{S}}
\end{array}\right]^{T}, \\
\mathbf{p}^{\text {Slave } \mid \text { Master=bad }} & =\left[\begin{array}{ll}
P_{\mathrm{g} \mid \mathrm{b}}^{\mathrm{S}} & P_{\mathrm{b} \mid \mathrm{b}}^{\mathrm{S}}
\end{array}\right]^{T} .
\end{aligned}
$$

$P_{i \mid j}^{S}$ defines the probability of the slave state $i$ in case of the master state is $j$.

Calculation of Probabilities for Joint States and Slave States (One Master, One Slave). From the master probabilities and conditional slave probabilities the combined probability vector $\mathbf{p}^{\text {joint }}$ is calculated:

$$
\mathbf{p}^{\text {joint }}=\left[\begin{array}{c}
P_{\mathrm{gg}} \\
P_{\mathrm{gb}} \\
P_{\mathrm{bg}} \\
P_{\mathrm{bb}}
\end{array}\right]=\left[\begin{array}{c}
P_{\mathrm{g}}^{\mathrm{M}} \cdot P_{\mathrm{g} \mid \mathrm{g}}^{\mathrm{S}} \\
P_{\mathrm{g}}^{\mathrm{M}} \cdot P_{\mathrm{b} \mid \mathrm{g}}^{\mathrm{S}} \\
P_{\mathrm{b}}^{\mathrm{M}} \cdot P_{\mathrm{g} \mid \mathrm{b}}^{\mathrm{S}} \\
P_{\mathrm{b}}^{\mathrm{M}} \cdot P_{\mathrm{b} \mid \mathrm{b}}^{\mathrm{S}}
\end{array}\right] .
$$

Based on $\mathrm{p}^{\text {joint }}$ the state probabilities of the slave can be calculated finally:

$$
\mathbf{p}^{\text {Slave }}=\left[\begin{array}{c}
P_{\mathrm{g}}^{\mathrm{S}} \\
P_{\mathrm{b}}^{\mathrm{S}}
\end{array}\right]=\left[\begin{array}{l}
P_{\mathrm{gg}}+P_{\mathrm{bg}} \\
P_{\mathrm{gb}}+P_{\mathrm{bb}}
\end{array}\right] .
$$

Calculation of State Probabilities with One Master and Multiple Slaves. Analogously to the two-satellite case, the combined probability vector $\mathbf{p}^{\text {joint }}$ for multiple satellites (with $k$ satellites) is calculated from the master probabilities and multiple conditional slave probabilities:

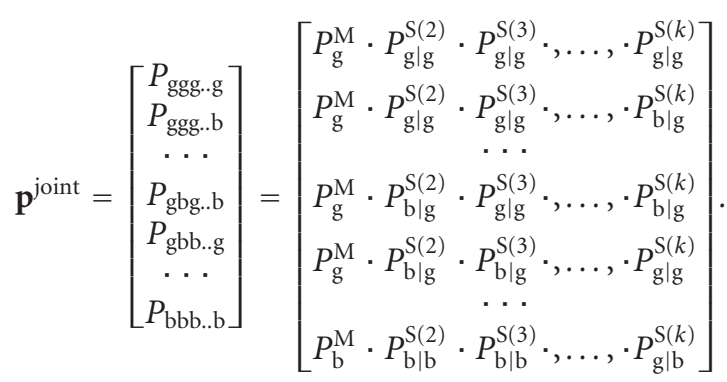

Correlation Coefficient between Master and Slave. Since only two states per satellite are assumed, the phi coefficient $\phi[11]$ 
is used to describe the correlation coefficient between two satellites:

$$
\phi=\frac{n_{11} \cdot n_{22}-n_{12} \cdot n_{21}}{\sqrt{\left(n_{1 X}\right)\left(n_{2 X}\right)\left(n_{X 1}\right)\left(n_{X 2}\right)}} .
$$

$n_{i j}$ is the number of samples where sequence 1 has state $i$ and sequence 2 has state $j$. For state modelling this formula can be adapted by using the joint state probabilities of two satellites:

$$
\rho_{\text {states }}=\frac{P_{\mathrm{gg}} \cdot P_{\mathrm{bb}}-P_{\mathrm{gb}} \cdot P_{\mathrm{bg}}}{\sqrt{\left(P_{\mathrm{gg}}+P_{\mathrm{gb}}\right)\left(P_{\mathrm{bb}}+P_{\mathrm{bg}}\right)\left(P_{\mathrm{gg}}+P_{\mathrm{bg}}\right)\left(P_{\mathrm{bb}}+P_{\mathrm{gb}}\right)}} .
$$

Correlation Coefficient between Two Slaves. The correlation coefficient between the slaves depends on the combined state probabilities $P_{i j}^{\prime}$ of the slaves:

$$
\begin{aligned}
& \rho_{\text {states, Slaves }}= \\
& \frac{P_{\mathrm{gg}}^{\prime} \cdot P_{\mathrm{bb}}^{\prime}-P_{\mathrm{gb}}^{\prime} \cdot P_{\mathrm{bg}}^{\prime}}{\sqrt{\left(P_{\mathrm{gg}}^{\prime}+P_{\mathrm{gb}}^{\prime}\right)\left(P_{\mathrm{bb}}^{\prime}+P_{\mathrm{bg}}^{\prime}\right)\left(P_{\mathrm{gg}}^{\prime}+P_{\mathrm{bg}}^{\prime}\right)\left(P_{\mathrm{bb}}^{\prime}+P_{\mathrm{gb}}^{\prime}\right)}} .
\end{aligned}
$$

The combined state probabilities $P_{i j}^{\prime}$ of the slaves can be calculated from the joint state probabilities of the whole system Master (1)-Slave (2)-Slave (3). According to (A.4) it holds:

$$
\begin{aligned}
\mathbf{p}^{\text {joint(Slave2, Slave3) }} & =\left[\begin{array}{l}
P_{\mathrm{gg}}^{\prime} \\
P_{\mathrm{gb}}^{\prime} \\
P_{\mathrm{bg}}^{\prime} \\
P_{\mathrm{bb}}^{\prime}
\end{array}\right]=\left[\begin{array}{l}
P_{\mathrm{ggg}}+P_{\mathrm{bgg}} \\
P_{\mathrm{ggb}}+P_{\mathrm{bgb}} \\
P_{\mathrm{gbg}}+P_{\mathrm{bbg}} \\
P_{\mathrm{gbb}}+P_{\mathrm{bbb}}
\end{array}\right] \\
& =\left[\begin{array}{l}
P_{\mathrm{g}}^{\mathrm{M}} \cdot P_{\mathrm{g} \mid \mathrm{g}}^{\mathrm{S}(2)} \cdot P_{\mathrm{g} \mid \mathrm{g}}^{\mathrm{S}(3)}+P_{\mathrm{b}}^{\mathrm{M}} \cdot P_{\mathrm{g} \mid \mathrm{b}}^{\mathrm{S}(2)} \cdot P_{\mathrm{g} \mid \mathrm{b}}^{\mathrm{S}(3)} \\
P_{\mathrm{g}}^{\mathrm{M}} \cdot P_{\mathrm{g} \mid \mathrm{g}}^{\mathrm{S}(2)} \cdot P_{\mathrm{b} \mid \mathrm{g}}^{\mathrm{S}(3)}+P_{\mathrm{b}}^{\mathrm{M}} \cdot P_{\mathrm{g} \mid \mathrm{b}}^{\mathrm{S}(2)} \cdot P_{\mathrm{b} \mid \mathrm{b}}^{\mathrm{S}(3)} \\
P_{\mathrm{g}}^{\mathrm{M}} \cdot P_{\mathrm{b} \mid \mathrm{g}}^{\mathrm{S}(2)} \cdot P_{\mathrm{g} \mid \mathrm{g}}^{\mathrm{S}(3)}+P_{\mathrm{b}}^{\mathrm{M}} \cdot P_{\mathrm{b} \mid \mathrm{b}}^{\mathrm{S}(2)} \cdot P_{\mathrm{g} \mid \mathrm{b}}^{\mathrm{S}(3)} \\
P_{\mathrm{g}}^{\mathrm{M}} \cdot P_{\mathrm{b} \mid \mathrm{g}}^{\mathrm{S}(2)} \cdot P_{\mathrm{b} \mid \mathrm{g}}^{\mathrm{S}(3)}+P_{\mathrm{b}}^{\mathrm{M}} \cdot P_{\mathrm{b} \mid \mathrm{b}}^{\mathrm{S}(2)} \cdot P_{\mathrm{b} \mid \mathrm{b}}^{\mathrm{S}(3)}
\end{array}\right] .
\end{aligned}
$$

By inserting (A.8) in (A.7) the correlation coefficient between the slaves $\rho_{\text {states, Slaves }}$ can be described as a function of the correlation coefficients between master and slave (2) and master and slave (3):

$$
\rho_{\text {states, Slaves }}=\rho_{\text {states, Master, Slave2 }} \cdot \rho_{\text {states, Master, Slave3. }}
$$

\section{Conflict of Interests}

The authors declare that they have no conflict of interests.

\section{Acknowledgments}

The state analysis in this paper is based on measurement data from the project MiLADY [9]. This project was funded by the ARTES 5.1 Programme of the Telecommunications and Integrated Applications Directorate of the European Space Agency.

\section{References}

[1] C. Loo, "A statistical model for a land mobile satellite link," in Proceedings of the International Conference on Communications (ICC '84), vol. 2 of Links for the future: Science, systems and services for communications, pp. 588-594, 1984.

[2] E. Lutz, D. Cygan, M. Dippold, F. Dolainsky, and W. Papke, "The land mobile satellite communication channel-recording, statistics, and channel model," IEEE Transactions on Vehicular Technology, vol. 40, no. 2, pp. 375-386, 1991.

[3] F. P. Fontán, M. Vázquez-Castro, C. E. Cabado, J. P. García, and E. Kubista, "Statistical modeling of the LMS channel," IEEE Transactions on Vehicular Technology, vol. 50, no. 6, pp. 15491567, 2001.

[4] R. Prieto-Cerdeira, F. Pérez-Fontán, P. Burzigotti, A. BoleaAlama nac, and I. Sanchez-Lago :, "Versatile two-state land mobile satellite channel model with first application to DVBSH analysis," International Journal of Satellite Communications and Networking, vol. 28, pp. 291-315, 2010.

[5] L. E. Bråten and T. Tjelta, "Semi-Markov multistate modeling of the land mobile propagation channel for geostationary satellites," IEEE Transactions on Antennas and Propagation, vol. 50, no. 12, pp. 1795-1802, 2002.

[6] M. Milojević, M. Haardt, E. Eberlein, and A. Heuberger, "Channel modeling for multiple satellite broadcasting systems," IEEE Transactions on Broadcasting, vol. 55, no. 4, pp. 705-718, 2009.

[7] D. Arndt, A. Ihlow, T. Heyn, A. Heuberger, R. PrietoCerdeira, and E. Eberlein, "State modelling of the land mobile propagation channel for dual-satellite systems," EURASIP Journal on Wireless Communications and Networking, vol. 2012, article 228, 2012.

[8] E. Lutz, "A Markov model for correlated land mobile satellite channels," International Journal of Satellite Communications, vol. 14, no. 4, pp. 333-339, 1996.

[9] E. Eberlein, A. Heuberger, and T. Heyn :, "Channel models for systems with angle diversity-the MiLADY project," in Proceedings of the ESA Workshop on Radiowave Propagation Models, Tools and Data for Space Systems, Noordwijk, the Netherlands, 2008.

[10] T. Heyn, E. Eberlein, D. Arndt et al., "Mobile satellite channel with angle diversity: the MiLADY project," in Proceedings of the 4th European Conference on Antennas and Propagation (EuCAP '10), Barcelona, Spain, April 2010.

[11] H. Cramer, Mathematical Methods of Statistics, Princeton University Press, Princeton, NJ, USA, 1946. 

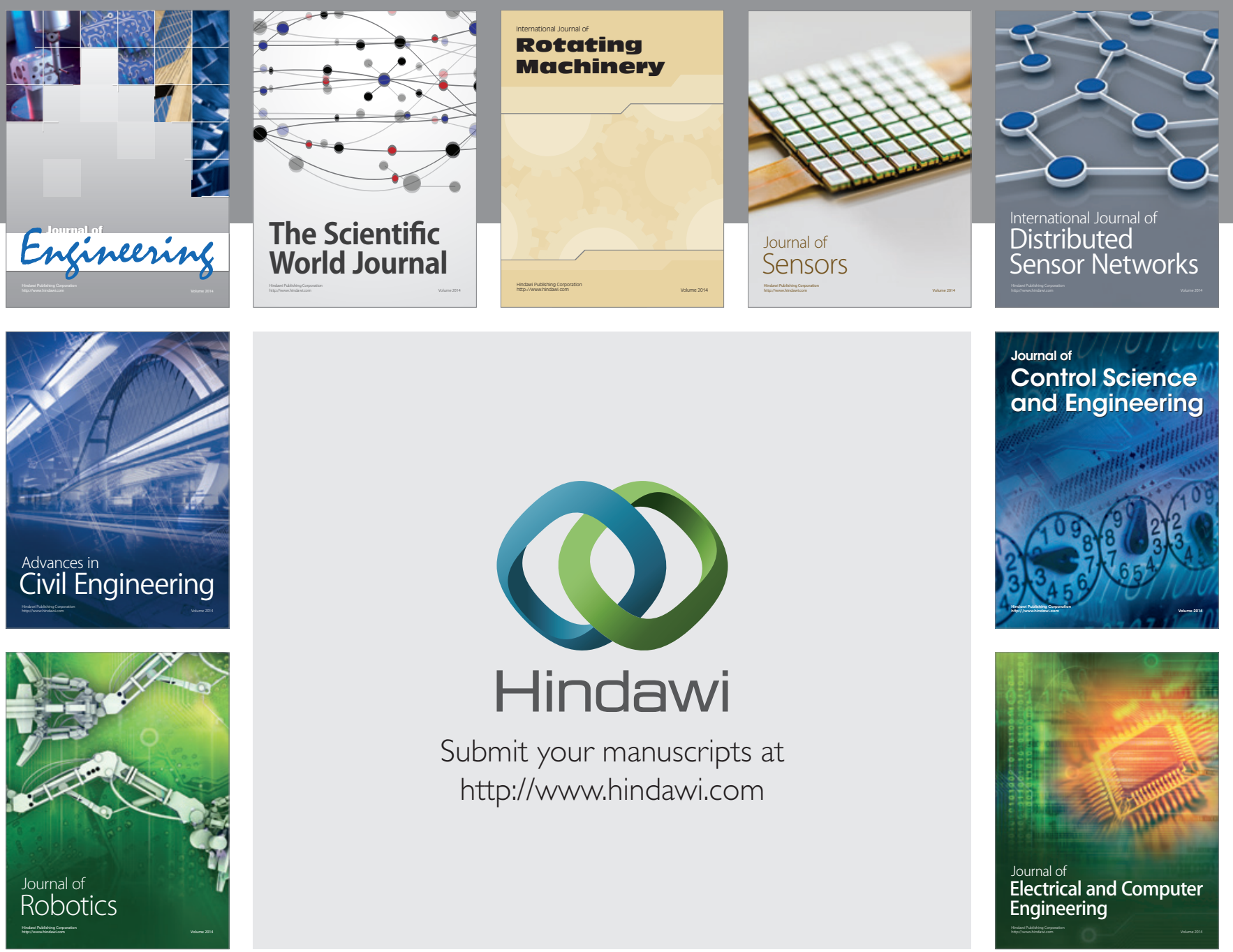

Submit your manuscripts at

http://www.hindawi.com
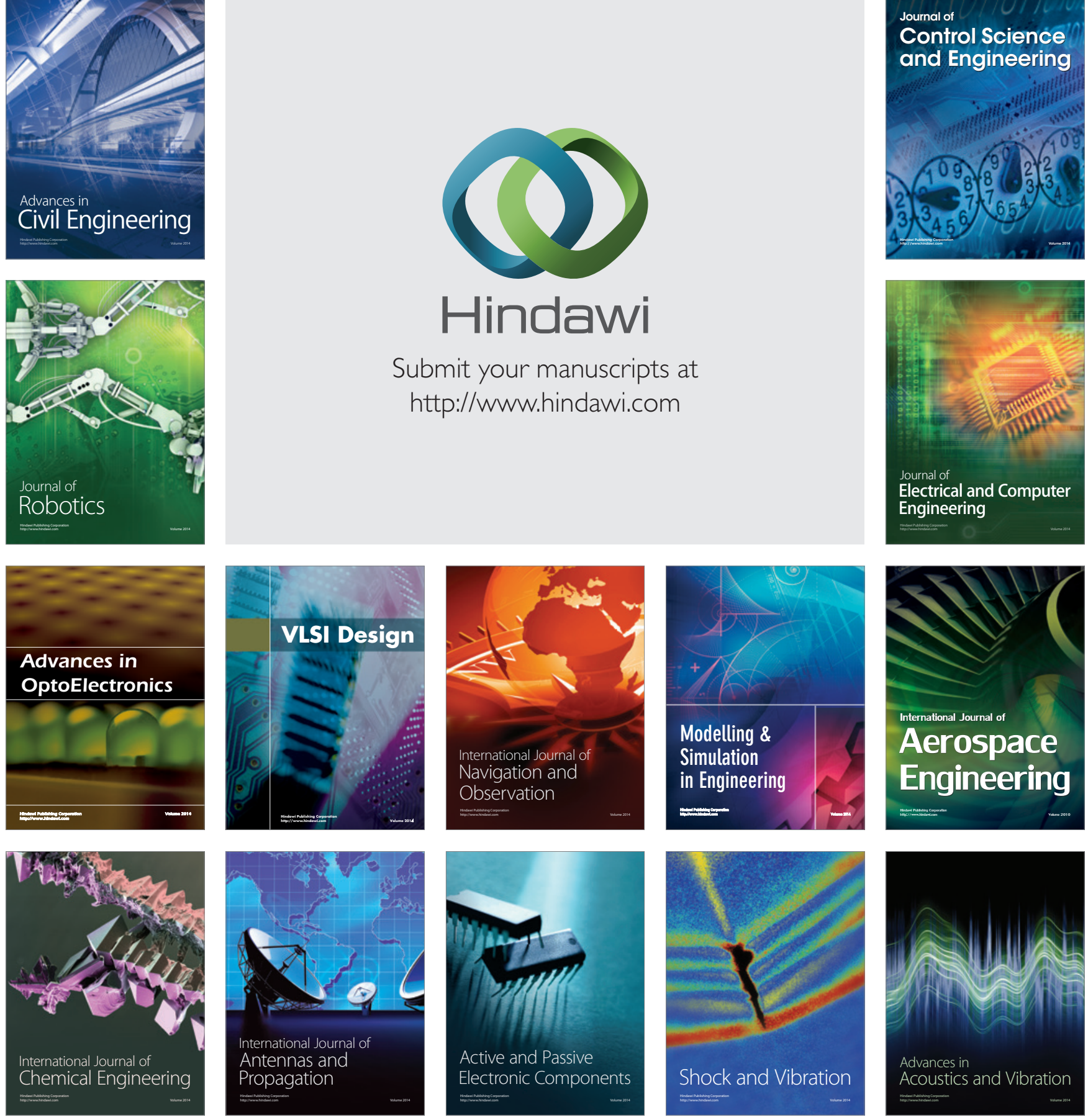